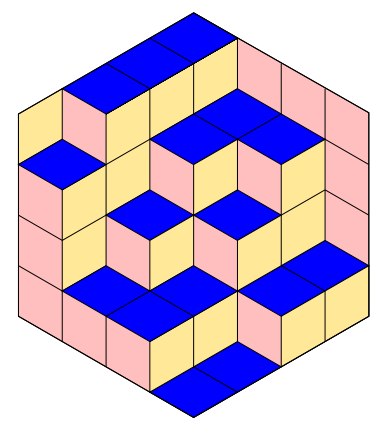

ALGEBRAIC COMBINATORICS

Svante Linusson, Samu Potka \& Robin Sulzgruber

On random shifted standard Young tableaux and 132-avoiding sorting networks

Volume 3, issue 6 (2020), p. 1231-1258.

<http://alco.centre-mersenne.org/item/ALCO_2020__3_6_1231_0>

(c) The journal and the authors, 2020.

Some rights reserved.

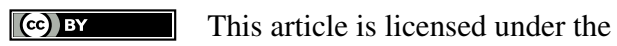

Creative Commons AtTribution 4.0 International LiCEnSE.

http://creativecommons.org/licenses/by/4.0/

Access to articles published by the journal Algebraic Combinatorics on the website http://alco.centre-mersenne.org/ implies agreement with the Terms of Use (http://alco.centre-mersenne.org/legal/).

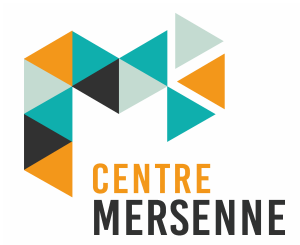

Algebraic Combinatorics is member of the Centre Mersenne for Open Scientific Publishing www.centre-mersenne.org 


\title{
On random shifted standard Young tableaux and 132-avoiding sorting networks
}

\author{
Svante Linusson, Samu Potka \& Robin Sulzgruber
}

\begin{abstract}
We study shifted standard Young tableaux (SYT). The limiting surface of uniformly random shifted SYT of staircase shape is determined, with the integers in the SYT as heights. This implies via properties of the Edelman-Greene bijection results about random 132-avoiding sorting networks, including limit shapes for trajectories and intermediate permutations. Moreover, the expected number of adjacencies in SYT is considered. It is shown that on average each row and each column of a shifted SYT of staircase shape contains precisely one adjacency.
\end{abstract}

\section{INTRODUCTION}

A shifted standard Young tableau (SYT) of staircase shape is an increasing filling of the shifted diagram of the partition $(n-1, \ldots, 2,1)$ with the integers $1,2, \ldots,\left(\begin{array}{l}n \\ 2\end{array}\right)$. See Figure 1a for an example and Section 2 for the exact definition. Shifted diagrams and tableaux are important combinatorial objects that appear in various contexts. In representation theory shifted Young diagrams correspond to projective characters of the symmetric group, and shifted tableaux lend themselves to being studied via RSK-type methods [32, 40]. In the theory of partially ordered sets, shifted diagrams alongside non-shifted Young diagrams and rooted trees form the three most interesting families of $d$-complete posets, which are in turn connected to fully commutative elements of Coxeter groups [41,28]. The most salient property of $d$-complete posets is the fact that their linear extensions (in our case shifted SYT) are enumerated by elegant product formulas involving hook lengths, see [28, 23]. Shifted diagrams also appear as order filters in the root poset of type $B_{n}$, and shifted SYT play an important role in the enumeration of reduced words of elements of the Coxeter group of type $B_{n}[15,18]$. Moreover, as is topical in this paper, shifted SYT are also relevant to the study of certain reduced words in the symmetric group. Recently, Elizalde and Roichman related shifted diagrams and tableaux to unimodal permutations [7].

The topics of this paper can be divided into three parts.

In Section 3 we study the surface obtained by viewing the integers in random SYT as heights. The study of limit phenomena for partitions and tableaux is an active field of research combining methods from combinatorics, probability theory and analysis. We refer to [30] for a general survey. Shifted objects have been treated as well, for example Ivanov [16] proves a central limit theorem for the Plancherel

Manuscript received 3rd May 2018, revised 24th February 2020, accepted 13th June 2020.

KEYwORDS. Shifted standard Young tableaux, random 132-avoiding sorting networks.

ACKnowledgements. The first two authors were supported by the Swedish Research Council, grant 621-2014-4780. 


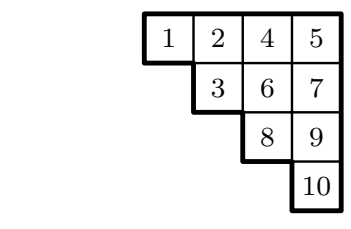

(a) A shifted SYT of staircase shape.

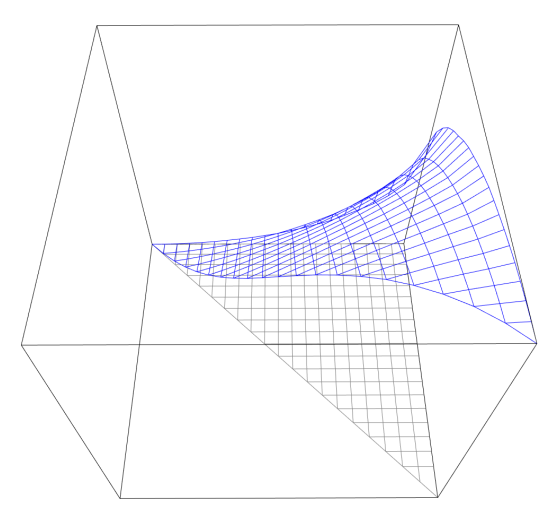

(b) The limit shape of uniformly random shifted SYT of staircase shape.

FIGURE 1

measure on shifted diagrams. In the present paper we determine the limiting surface for uniformly random shifted SYT of staircase shape, see Figure 1b and Theorem 3.7. The deduction of our results relies on a paper by Pittel and Romik [27] where the limit shape for random rectangular SYT is determined. In fact, we end up with the same variational problem, and the limit surface for shifted staircase SYT is half of the surface for square SYT. This analogy is in part explained by a combinatorial identity in Proposition 3.1 relating shifted and non-shifted tableaux. There are few shapes for which the limit surface has been explicitly described previously. As far as we know the only other case is that of staircase SYT, where again the same limit surface appears, but cut along a different diagonal [1]. However, it was pointed out to us by one of the referees that Biane [2] provides a general method for computing such limits for SYT. Recently, Matsumoto and Śniady developed an analogous framework for shifted SYT which, combined with the results of [2] and [27], allowed them to partially recover Theorem 3.7 in [22, Sec. 8.4]. Results of this type have applications in other fields of mathematics such as geometric complexity theory [26].

Secondly, we study 132-avoiding sorting networks, which are by definition reduced words $w_{1} \ldots w_{\left(\begin{array}{c}n \\ 2\end{array}\right)}$ of the reverse permutation such that $s_{w_{1}} \cdots s_{w_{k}}$ is 132 -avoiding for any $1 \leqslant k \leqslant\left(\begin{array}{l}n \\ 2\end{array}\right)$. These objects have received considerable recent interest and also appear in different guises, for example as chains of maximum length in the Tamari lattice [3]. Fishel and Nelson [9] showed that 132-avoiding sorting networks are in bijection with shifted SYT of staircase shape via the Edelman-Greene correspondence. This has been rediscovered several times [36, 5, 20]. They are also in bijection with reduced words of the signed permutation $(-(n-1),-(n-2), \ldots,-1)$ via the shift $s_{i} \mapsto s_{i-1}$ as was remarked in [36, Sec. 1.3]. In Sections 4 and 5 the Edelman-Greene bijection is used to transfer the limit shape of shifted SYT to determine the limit shapes of intermediate permutations (Theorem 4.2) and trajectories (Theorem 5.4) in random 132-avoiding sorting networks. These results are motivated by a remarkable paper of Angel, Holroyd, Romik and Virág [1] that contains a number of tantalising conjectures about random sorting networks, now proven by Dauvergne [4]. See Section 2 for a description of some of them. Our results are a parallel to their (former) conjectures restricted to a subclass of random sorting networks. The 132-avoiding sorting networks are much easier to treat than the general case. The main reason for 
this is that the Edelman-Greene bijection simplifies significantly when it is restricted to 132-avoiding sorting networks.

We remark that the limit surface for shifted SYT of staircase shape contains complete information on the limit surface for SYT of square shape. This suggests the perhaps less intuitive idea that the relatively small subset of 132-avoiding sorting networks contains a lot of information on random sorting networks in general.

The third set of results is obtained in Section 6 and concerns patterns in 132avoiding sorting networks. We first observe that adjacencies in a shifted SYT (that is, integers $i$ and $i+1$ in neighbouring cells) translate directly to adjacencies in a 132avoiding sorting network (that is, $j$ and $j+1$ next to each other in the reduced word). Corollary 6.5 asserts that the expected number of adjacencies in each column and each row in a shifted SYT of staircase shape is exactly 1 . The proof uses promotion and evacuation techniques very similar to the methods used by Schilling, Thiéry, White and Williams [36] to derive results on Yang-Baxter moves (that is, patterns of the form $j(j \pm 1) j$ ) in 132-avoiding sorting networks. Related results on general sorting networks are due to Reiner [29] and Tenner [43].

\section{BACKGROUND}

In this section we fix notation and review some facts about partitions, tableaux and random sorting networks.

For $n \in \mathbb{N}$ let $[n]=\{1, \ldots, n\}$. Throughout this paper we denote $N=\left(\begin{array}{l}n \\ 2\end{array}\right)$.

2.1. Partitions and tableaux. A partition is a weakly decreasing sequence $\lambda=$ $\left(\lambda_{1}, \ldots, \lambda_{n}\right)$ of positive integers. If a partition is strictly decreasing it is called strict. The sum $\sum \lambda_{i}$ is called the size of the partition $\lambda$ and is denoted by $|\lambda|$. The number of entries $\lambda_{i}$ is called the length of the partition and is denoted by $\ell(\lambda)$. Define the staircase partition as

$$
\Delta_{n}=(n-1, \ldots, 2,1) .
$$

The Young diagram of a partition $\lambda$ is defined as the set

$$
\lambda^{\mathrm{dg}}=\left\{(i, j): i \in[\ell(\lambda)], j \in\left[\lambda_{i}\right]\right\} .
$$

The elements $(i, j)$ are indexed with matrix notation and typically referred to as cells of $\lambda$. The conjugate partition $\lambda^{\prime}$ of the partition $\lambda$ is the partition corresponding to the Young diagram $\left\{(j, i):(i, j) \in \lambda^{\mathrm{dg}}\right\}$. Given a strict partition $\lambda$ we also define its shifted Young diagram as

$$
\lambda^{\mathrm{sh}}=\left\{(i, j+i-1): i \in[\ell(\lambda)], j \in\left[\lambda_{i}\right]\right\} .
$$

Thus the shifted Young diagram is obtained from the normal Young diagram by shifting rows to the right. These definitions are illustrated in Figure 2.
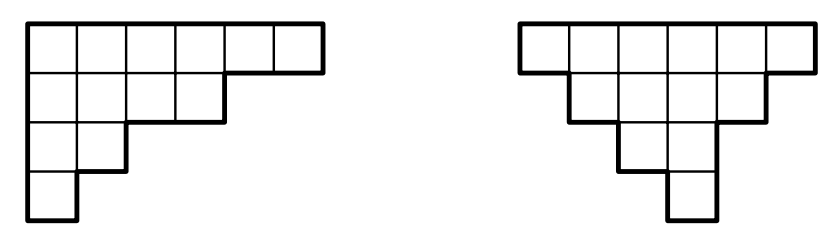

Figure 2. The Young diagram $\lambda^{\mathrm{dg}}$ (left) and the shifted Young diagram $\lambda^{\text {sh }}$ (right) of the strict partition $\lambda=(6,4,2,1)$ drawn in English convention, that is, $(1,1)$ is the top left cell. We have $|\lambda|=13$, $\ell(\lambda)=4$ and $\lambda^{\prime}=(4,3,2,2,1,1)$. 
Let $u=(i, j)$ be a cell in the Young diagram of a partition $\lambda$. The hook-length of $u$, denoted by $h_{\lambda}(u)$, is defined as the number of cells in the same row as $u$ that lie to the right of $u$ plus the number of cells in the same column as $u$ that lie weakly below $u$ (that is, including $u$ itself). Thus, $h_{\lambda}(u)=\lambda_{i}-j+\lambda_{j}^{\prime}-i+1$. Given a cell $u=(i, j)$ in the shifted Young diagram of a strict partition, we define its shifted hook-length $h_{\lambda}^{\mathrm{sh}}(u)$ as the number of cells in the same row as $u$ that lie to the right of $u$, or in the same column and weakly below $u$, plus the number of cells in the $(i+j)$-th row of the shifted Young diagram. See Figure 3.
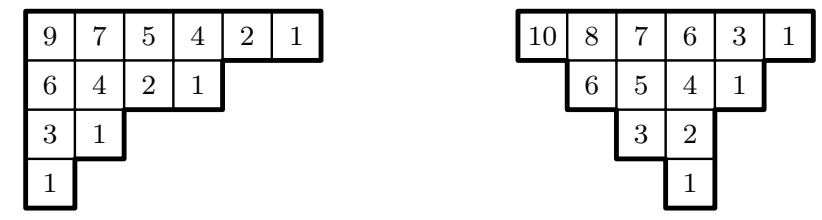

Figure 3. The (shifted) Young diagram of $\lambda=(6,4,2,1)$ with the (shifted) hook-lengths filled into each cell.

Given a cell $u=(i, j) \in \mathbb{Z}^{2}$, define the north, east, south, and west neighbour of $u$ as

$$
\mathbf{n} u=(i-1, j), \quad \mathbf{e} u=(i, j+1), \quad \mathbf{s} u=(i+1, j) \quad \text { and } \quad \mathbf{w} u=(i, j-1),
$$

respectively. A tableau of shape $\lambda^{\mathrm{dg}}$ is a map $T: \lambda^{\mathrm{dg}} \rightarrow \mathbb{Z}$. A tableau $T$ is called a standard Young tableau (SYT) if $T: \lambda^{\mathrm{dg}} \rightarrow[n]$ is a bijection and $T(u)<T(\mathbf{e} u)$ and $T(u)<T(\mathbf{s} u)$ whenever the respective cells lie in $\lambda^{\mathrm{dg}}$. Similarly a shifted standard Young tableau of shape $\lambda^{\text {sh }}$ is a bijection $T: \lambda^{\text {sh }} \rightarrow[n]$ such that $T(u)<T(\mathbf{e} u)$ and $T(u)<T(\mathbf{s} u)$ whenever the respective cells lie in the shifted Young diagram $\lambda^{\text {sh }}$. Let

$$
\mathcal{T}_{n}=\left\{T: T \text { is a shifted SYT of shape } \Delta_{n}^{\mathrm{sh}}\right\} .
$$

For example, Figure 1a shows a shifted standard Young diagram of shape $\Delta_{5}^{\mathrm{sh}}$.

The number of SYT is given be the following nice product formula.

THEOREM 2.1 (Hook-length formula $[11,44])$. Let $\lambda$ be a partition. Then the number of SYT of shape $\lambda^{\mathrm{dg}}$ is given by

$$
f_{\lambda}=\frac{|\lambda| !}{\prod_{u \in \lambda^{\mathrm{dg}}} h_{\lambda}(u)} .
$$

If $\lambda$ is strict then the number of shifted SYT of shape $\lambda^{\text {sh }}$ is given by

$$
f_{\lambda}^{\mathrm{sh}}=\frac{|\lambda| !}{\prod_{u \in \lambda^{\mathrm{sh}}} h_{\lambda}^{\mathrm{sh}}(u)} .
$$

The literature offers a variety of proofs of different flavours for Theorem 2.1, for example using hook-walks $[13,31]$ or by means of jeu de taquin $[24,8]$.

2.2. Random 132-AVOIDING SORTING NETwORKs. The reader is referred to [17] for background on pattern avoidance in permutations.

For $i \in[n-1]$ let $s_{i}=(i, i+1)$ denote the $i$-th adjacent transposition. The reverse permutation $w_{0} \in \mathfrak{S}_{n}$ is defined by $w_{0}(i)=n-i+1$ for $i \in[n]$. A reduced word of $w_{0}$ is a word $w=w_{1} \cdots w_{N}$ in the alphabet $[n-1]$ such that $w_{0}=s_{w_{1}} \cdots s_{w_{N}}$.

We will adopt the convention that multiplying a permutation by $s_{i}$ from the right interchanges the numbers in positions $i$ and $i+1$ (or swaps columns $i$ and $i+1$ of the permutation matrix, see below). This means equivalently that multiplying by $s_{i}$ from the left interchanges the positions of the values $i$ and $i+1$ (or swaps rows $i$ and $i+1$ of the permutation matrix). As an example consider the word $w=1213$, 
corresponding to composing $s_{1} s_{2} s_{1} s_{3}$, which yields the permutation 3241 . In terms of permutation matrices, we have

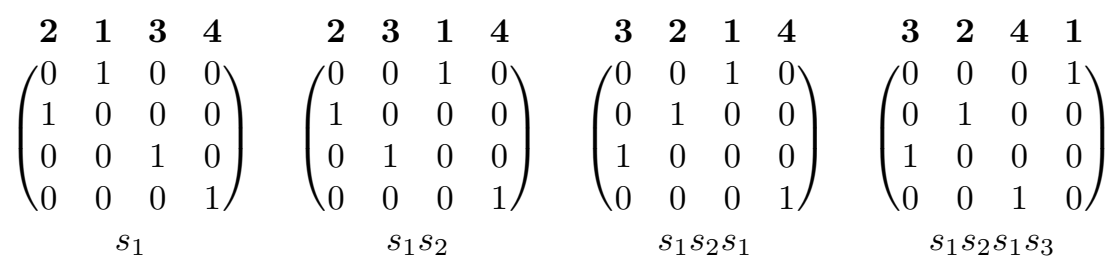

where we can see that multiplying by $s_{i}$ from the right corresponds to swapping the columns $i$ and $i+1$.

Angel, Holroyd, Romik and Virág introduced $n$-element random sorting networks in [1] as the set of reduced words of the reverse permutation $w_{0} \in \mathfrak{S}_{n}$ equipped with the uniform probability measure. In the same paper, Angel et al. pose several striking conjectures about random sorting networks, which have now been proven by Dauvergne [4].

- Suppose $w=w_{1} \ldots w_{N}$ is a sorting network. Then $w_{1} \ldots w_{k}$ defines the $i n$ termediate permutation $\sigma_{k}=s_{w_{1}} \cdots s_{w_{k}} \in \mathfrak{S}_{n}$ for all $k \in[N]$. One of the consequences of [4, Thm. 2] (previously [1, Conj. 2]) is that asymptotically the permutation matrices corresponding to the intermediate configurations coming from random sorting networks are supported on a family of ellipses. In other words, their 1s occur inside an elliptic region of the matrix. In particular, at half-time the permutation matrix is supported on a disc. Figure 4 provides an illustration.

- For $0 \leqslant \alpha \leqslant 1$, [4, Thm. 1] (previously [1, Conj. 1]) states that the scaled trajectories defined by

$$
f_{w, i}(\alpha)=\frac{2 \sigma_{\alpha N}^{-1}(i)}{n}-1
$$

for $\alpha N \in \mathbb{Z}$, and by linear interpolation otherwise, converge to random sine curves. See Figure 5.

- The permutahedron is an embedding of $\mathfrak{S}_{n}$ into $\mathbb{R}^{n}$ defined by

$$
\sigma \mapsto\left(\sigma^{-1}(1), \ldots, \sigma^{-1}(n)\right) .
$$

Every permutation $\sigma \in \mathfrak{S}_{n}$ lies on the sphere

$\mathbb{S}^{n-2}=\left\{z \in \mathbb{R}^{n}: \sum_{i=1}^{n} z_{i}=\frac{n(n+1)}{2}\right.$ and $\left.\sum_{i=1}^{n} z_{i}^{2}=\frac{n(n+1)(2 n+1)}{6}\right\}$.

Random sorting networks correspond to paths on the permutahedron. The strongest theorem, [4, Thm. 4] (previously [1, Conj. 3]), which implies both of the previous ones, states that these paths are close to great circles in $\mathbb{S}^{n-2}$

This paper considers similar questions restricted to 132-avoiding sorting networks, that is, those reduced words $w_{1} \ldots w_{N}$ of the reverse permutation in $\mathfrak{S}_{n}$ such that $s_{w_{1}} \cdots s_{w_{k}}$ is 132 -avoiding for all $k \in[N]$. With a random 132-avoiding sorting network we will refer to uniform distribution on all 132-avoiding sorting networks of a fixed length. Understanding them is easier than random sorting networks since the Edelman-Greene correspondence restricted to 132-avoiding sorting networks has a much simpler description than the general bijection. This is discussed next.

The connection between 132-avoiding sorting networks and shifted SYT is the following. Let $w=w_{1} \ldots w_{N}$ be a 132-avoiding sorting network. Then, for $k \in[N]$, define a SYT $Q_{w_{1} \ldots w_{k}}$ by letting its $j$-th column consist of the indices $m \in[k]$ such 

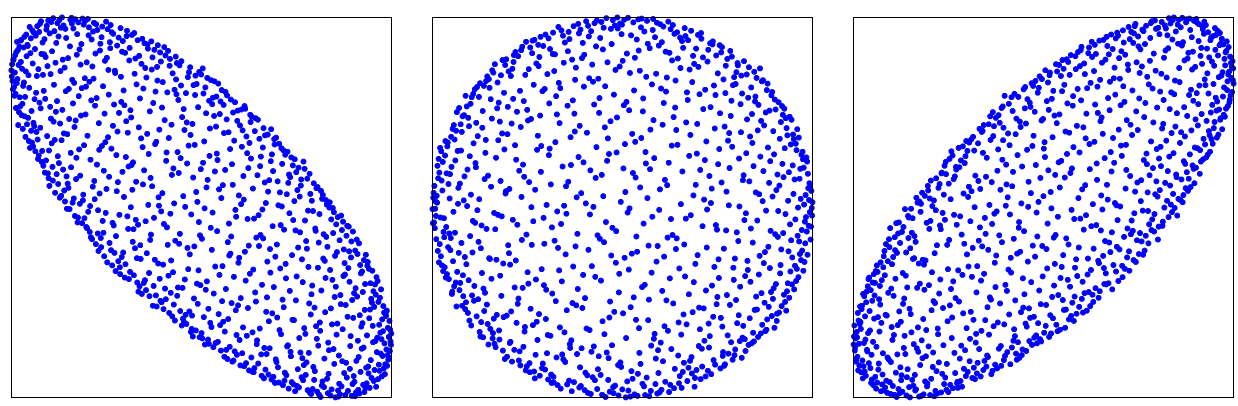

FIGURE 4. The intermediate permutation matrices of $\sigma_{\lfloor\alpha N\rfloor}$ of a 1000-element random sorting network at times $\alpha=\frac{1}{4}, \frac{1}{2}$ and $\frac{3}{4}$.

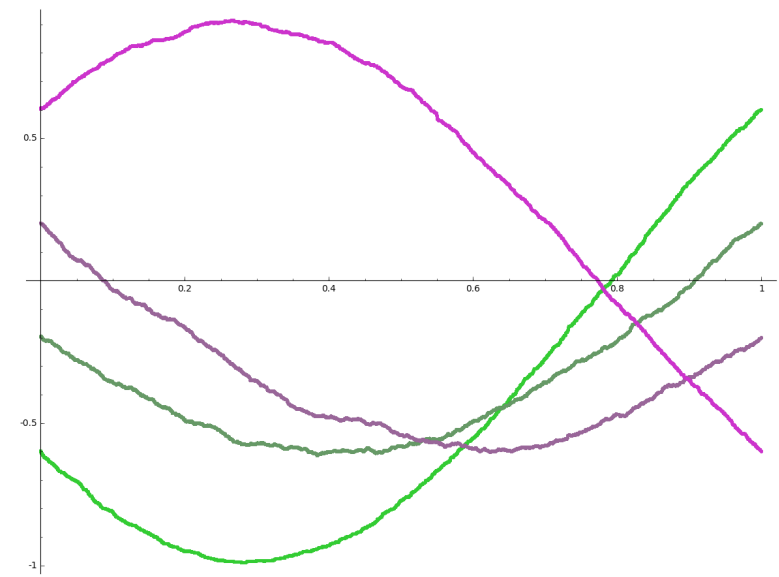

Figure 5. The scaled trajectories of the elements 200, 400, 600 and 800 in a 1000-element random sorting network.

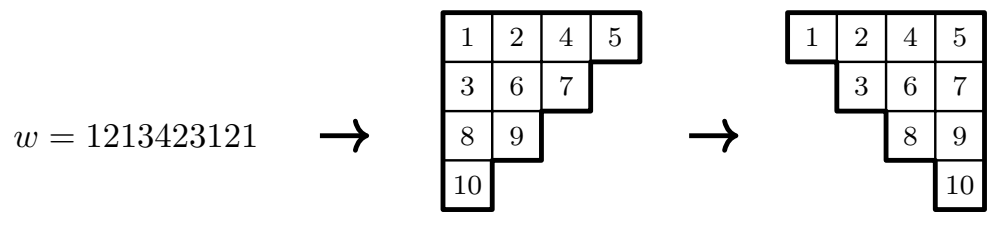

Figure 6. The Edelman-Greene correspondence for a 5-element 132-avoiding sorting network.

that $w_{m}=j$. Furthermore, define a shifted SYT $Q_{w_{1} \ldots w_{k}}$ by shifting the rows of $Q_{w_{1} \ldots w_{k}}$. Figure 6 shows an example.

TheOREM 2.2 ([9, Thm. 3.3 and Thm. 4.6]). For all $n \in \mathbb{N}$, the map $w \mapsto Q_{w}$ is a bijection from n-element 132-avoiding sorting networks to shifted $S Y T$ of shape $\Delta_{n}^{\text {sh }}$. The map $w \mapsto Q_{w}$ agrees with the restriction of the Edelman-Greene correspondence to 132-avoiding sorting networks.

The same bijection was also described in [20], in [36, Fig. 4] in terms of heaps, and in [5, Prop. 5.2] in terms of descent sets. 
We conclude this section with two more facts about 132-avoiding sorting networks, see [20] for proofs. First, reversing a sorting network preserves the property of being 132-avoiding.

Proposition 2.3. A reduced word $w_{1} \ldots w_{N}$ is a 132-avoiding sorting network if and only if $w_{N} \ldots w_{1}$ is.

Proof. This can be seen by flipping $Q_{w}$ about the anti-diagonal and replacing each entry $k$ by $N+1-k$.

Secondly, the set of 132-avoiding and 312-avoiding sorting networks coincide.

Proposition 2.4 ([20, Prop. 3.10]). A reduced word $w$ is a 132-avoiding sorting network if and only if $w$ is a 312-avoiding sorting network.

\section{The Limit SHAPE}

In this section we derive a limit shape for random shifted SYT of staircase shape. We may interpret a shifted SYT $T \in \mathcal{T}_{n}$ as the graph of a function

$$
L_{T}:\left\{(x, y) \in \mathbb{R}^{2}: 0<x<y<1\right\} \rightarrow \mathbb{R}_{\geqslant 0}
$$

by viewing the entries as heights

$$
L_{T}(x, y)=\frac{1}{N} T(\lceil n x\rceil,\lceil n y\rceil) .
$$

Our main result, Theorem 3.7, states that by this choice of scaling, the functions $L_{T^{(n)}}$ converge with probability 1 to the surface depicted in Figure 1b, where each $T^{(n)} \in \mathcal{T}_{n}$ is chosen uniformly at random. The proof of Theorem 3.7 relies heavily on the work of Pittel and Romik [27].

Recall our conventions from Section 2.1. Given a strict partition $\lambda$ define a partition $\Lambda$ by letting its Young diagram equal

$$
\Lambda^{\mathrm{dg}}=\left\{(i, j+1):(i, j) \in \lambda^{\mathrm{sh}}\right\} \cup\left\{(j, i):(i, j) \in \lambda^{\mathrm{sh}}\right\} .
$$

It is easy to see that this really is the Young diagram of a partition. See Figure 7 . We call $\Lambda$ the double of $\lambda$. The diagram of $\Lambda$ is at times, for example in [21], denoted by $D(\lambda)$. The motivation for this definition is the fact that shifted hook-lengths of the cells in $\lambda^{\text {sh }}$ correspond to hook-lengths of the corresponding cells in $\Lambda^{\mathrm{dg}}$.

The following proposition is not new and can be obtained by extracting coefficients from an identity of symmetric functions found in [21, Chap. III, Sec. 8, Example 9.(b)]. We include a proof nevertheless since it is short and needs no additional terminology.

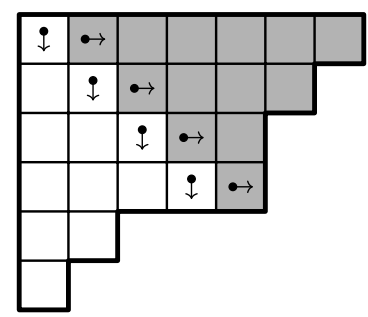

Figure 7 . The double $\Lambda=(7,6,5,5,2,1)$ of the strict partition $\lambda=(6,4,2,1)$.

Proposition 3.1. Let $\lambda$ be a strict partition and $\Lambda$ its double. Then

$$
f_{\Lambda}=\left(f_{\lambda}^{\mathrm{sh}}\right)^{2} \cdot\left(\begin{array}{c}
2|\lambda| \\
|\lambda|
\end{array}\right) \cdot 2^{-\ell(\lambda)} .
$$


Proof. We show that

$$
\prod_{u \in \Lambda^{\mathrm{dg}}} h_{\Lambda}(u)=2^{\ell(\lambda)}\left(\prod_{u \in \lambda^{\mathrm{sh}}} h_{\lambda}^{\mathrm{sh}}(u)\right)^{2} .
$$

This is obvious if $\lambda$ is a staircase and $\Lambda$ is a rectangle. In this case

$$
h_{\Lambda}(i, i)=2 h_{\Lambda}(i, \ell(\lambda)+1)=2 h_{\lambda}^{\mathrm{sh}}(i, \ell(\lambda)),
$$

as in Figure 8a, and

$$
h_{\Lambda}(i, j)=h_{\Lambda}(j, i)=h_{\lambda}^{\mathrm{sh}}(i, j-1),
$$

when $i<j \leqslant \ell(\lambda)$ as in Figure 8b.

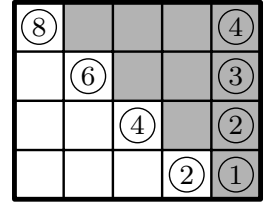

(a) The hook-lengths on the diagonal and in the last column of $\Lambda$ satisfy $h_{\Lambda}(i, i)=$ $2 h_{\Lambda}(i, \ell(\lambda)+1)=2 h_{\lambda}^{\mathrm{sh}}(i, \ell(\lambda))$.

\begin{tabular}{|l|l|l|l|l|}
\hline & 7 & 6 & 5 & \\
\hline 7 & & 5 & 4 & \\
\hline 6 & 5 & & 3 & \\
\hline 5 & 4 & 3 & & \\
\hline
\end{tabular}

(b) The off-diagonal hook-lengths to the left of the last column of $\Lambda$ satisfy $h_{\Lambda}(i, j)=$ $h_{\Lambda}(j, i)=h_{\lambda}^{\mathrm{sh}}(i, j-1)$.

FiguRE 8. Matching the hook-lengths of a staircase partition $\lambda$ and its double $\Lambda$, a rectangle.

The identity in (2) follows inductively as it is easy to verify that (3) and (4) are preserved when a cell $(i, k)$ with $\ell(\lambda)<k$ is added to $\lambda^{\text {sh }}$. Adding a cell to row $i$ increases $h_{\lambda}^{\text {sh }}(i, \ell(\lambda))$ by 1 and $h_{\Lambda}(i, i)$ by 2 , hence the equality in (3) still holds. Moreover, since $(k, i)$ and $(i, k+1)$ are added to $\Lambda^{\mathrm{dg}}$, the equality in $(4)$ is preserved. For the remaining cells we have $h_{\Lambda}\left(i^{\prime}, j^{\prime}\right)=h_{\Lambda}\left(j^{\prime}-1, i^{\prime}\right)=h_{\lambda}^{\mathrm{sh}}\left(i^{\prime}, j^{\prime}-1\right)$ for $i^{\prime} \leqslant \ell(\lambda)<j^{\prime}$. See Figure 9 .

The claim then follows from the hook-length formula (Theorem 2.1).
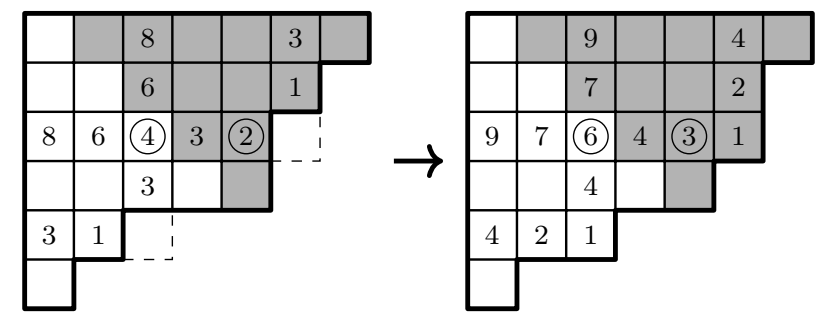

Figure 9. The effect of adding a cell on the hook-lengths in a shifted diagram and its double.

The following two results provide an estimate for the probability that a fixed subdiagram $\lambda^{\text {sh }}$ of size $k$ of the shifted staircase contains precisely the entries $1, \ldots, k$ in a shifted SYT of shape $\Delta_{n}^{\text {sh }}$ chosen uniformly at random.

Let $m, n \in \mathbb{N}, \square=\left(n^{m}\right)$, and $\lambda$ be a partition. If $\lambda^{\mathrm{dg}} \subseteq \square^{\text {dg }}$ define a partition $\square \backslash \lambda$ by setting

$$
(\square \backslash \lambda)^{\mathrm{dg}}=\left\{(m-i+1, n-j+1):(i, j) \in \square^{\mathrm{dg}} \backslash \lambda^{\mathrm{dg}}\right\} .
$$


This is a $180^{\circ}$ rotation of the skew diagram of $\square \backslash \lambda$. Moreover, if $\lambda$ is strict and $\lambda^{\text {sh }} \subseteq \Delta_{n}^{\text {sh }}$, define a strict partition $\Delta_{n} \backslash \lambda$ by setting

$$
\left(\Delta_{n} \backslash \lambda\right)^{\mathrm{sh}}=\left\{(n-j, n-i):(i, j) \in \Delta_{n}^{\mathrm{sh}} \backslash \lambda^{\mathrm{sh}}\right\},
$$

which is the reflection of $\Delta_{n}^{\mathrm{sh}} \backslash \lambda^{\mathrm{sh}}$ through the diagonal $i=j$. See Figure 10 .

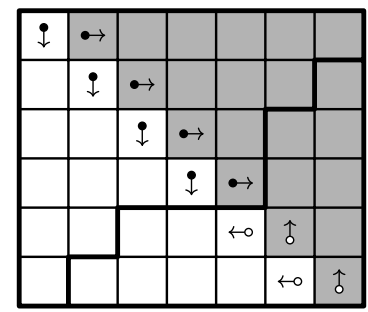

Figure 10. The partition $\square_{7}=\left(7^{6}\right)$. The shifted diagram of $\lambda=$ $(6,4,2,1)$ is in grey in the upper left part, which is the diagram of $\Lambda=(7,6,5,5,2,1)$. The diagram of $\square_{7} \backslash \Lambda=(6,5,2,2,1)$ is a $180^{\circ}$ rotation of the lower right part. The shifted diagram of $\Delta_{7} \backslash \lambda=(5,3)$ is the reflection through the diagonal $i=j$ of the grey region in the lower right part. The partition $\square_{7} \backslash \Lambda=(6,5,2,2,1)$ is the double of $\Delta_{7} \backslash \lambda$.

LEMMA 3.2. Let $n \in \mathbb{N}, \lambda$ be a strict partition of size $k$ with $\lambda_{1}<n$, and let $\mathbb{P}_{n}$ denote the uniform probability measure on $\mathcal{T}_{n}$. Then

$$
\mathbb{P}_{n}\left(T \in \mathcal{T}_{n}: T\left(\lambda^{\mathrm{sh}}\right)=[k]\right)=\left(\begin{array}{c}
N \\
k
\end{array}\right)^{-1} \cdot \sqrt{\left(\begin{array}{c}
2 N \\
2 k
\end{array}\right) \frac{f_{\Lambda} \cdot f_{\square_{n} \backslash \Lambda}}{f_{\square_{n}}}},
$$

where $\Lambda$ and $\square_{n}$ are the doubles corresponding to $\lambda$ and $\Delta_{n}$ respectively.

Proof. The left hand side of (5) is equal to

$$
\mathbb{P}_{n}\left(T \in \mathcal{T}_{n}: T\left(\lambda^{\mathrm{sh}}\right)=[k]\right)=\frac{f_{\lambda}^{\mathrm{sh}} \cdot f_{\Delta_{n} \backslash \lambda}^{\mathrm{sh}}}{f_{\Delta_{n}}^{\mathrm{sh}}} .
$$

It is not difficult to see that $\square_{n} \backslash \Lambda$ is the double of $\Delta_{n} \backslash \lambda$. See Figure 10. Thus (6) can be computed by means of Proposition 3.1 above.

The right hand side of (5) is similar to [27, Eq. (7)]. This allows us to prove an analogue of $\left[27\right.$, Lem. 1] for the shifted staircase. Note that $\square_{n}$ denotes an $n \times n$ square in [27], whereas here it is an $(n-1) \times n$ rectangle.

We now describe scaled and rotated boundary functions of partitions. We use the same conventions as Pittel and Romik. Fix $n$ and let $\lambda$ be a partition with $|\lambda|=k$. Define a function $\gamma_{\lambda}: \mathbb{R}_{>0} \rightarrow \mathbb{R}_{\geqslant 0}$ by

$$
\gamma_{\lambda}(x)=\frac{1}{n} \lambda_{\lceil n x\rceil}
$$

where by convention $\lambda_{i}=0$ for $i>\ell(\lambda)$. See Figure 11. It is often more convenient to work with rotated coordinates

$$
u=\frac{x-y}{\sqrt{2}}, \quad v=\frac{x+y}{\sqrt{2}} .
$$

Define $g_{\lambda}: \mathbb{R} \rightarrow \mathbb{R}_{\geqslant 0}$ by

$$
g_{\lambda}(u)=\sup \left\{v: v=|u| \text { or } x(u, v)>0, y(u, v) \leqslant \gamma_{\lambda}(x(u, v))\right\}
$$



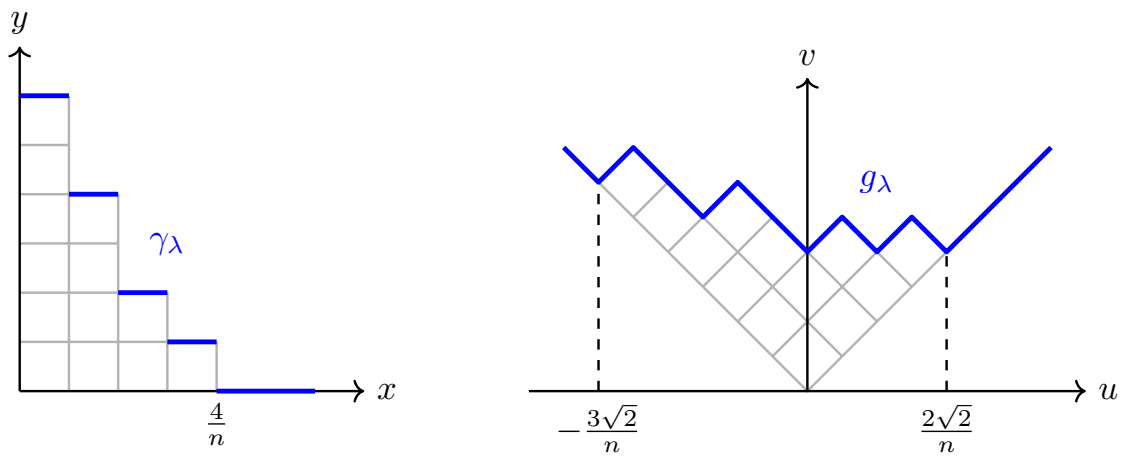

Figure 11. The functions $\gamma_{\lambda}$ and $g_{\lambda}$ for the partition $(6,4,2,1)$.

where $x(u, v)$ and $y(u, v)$ are given by the inverse of the transformation in Equation (7). The function $g_{\lambda}$ is just a rotated version of the function $\gamma_{\lambda}$, however, it has the advantage of being 1-Lipschitz while $\gamma_{\lambda}$ is only non-increasing. Note that

$$
\int_{0}^{\infty} \gamma_{\lambda}(x) \mathrm{d} x=\int_{\mathbb{R}} g_{\lambda}(u)-|u| \mathrm{d} u=\frac{k}{n^{2}} .
$$

If the partition $\lambda$ is strict we define $G_{\lambda}: \mathbb{R}_{\leqslant 0} \rightarrow \mathbb{R}_{\geqslant 0}$ by

$$
G_{\lambda}(u)=\sup \left\{v: v=|u| \text { or } x>0, y \leqslant \gamma_{\lambda}(x)+\frac{\lceil n x\rceil-1}{n}\right\},
$$

where implicitly $x=x(u, v)$ and $y=y(u, v)$. The function $G_{\lambda}$ describes the boundary of the scaled shifted Young diagram $\lambda^{\text {sh }}$. See Figure 12.
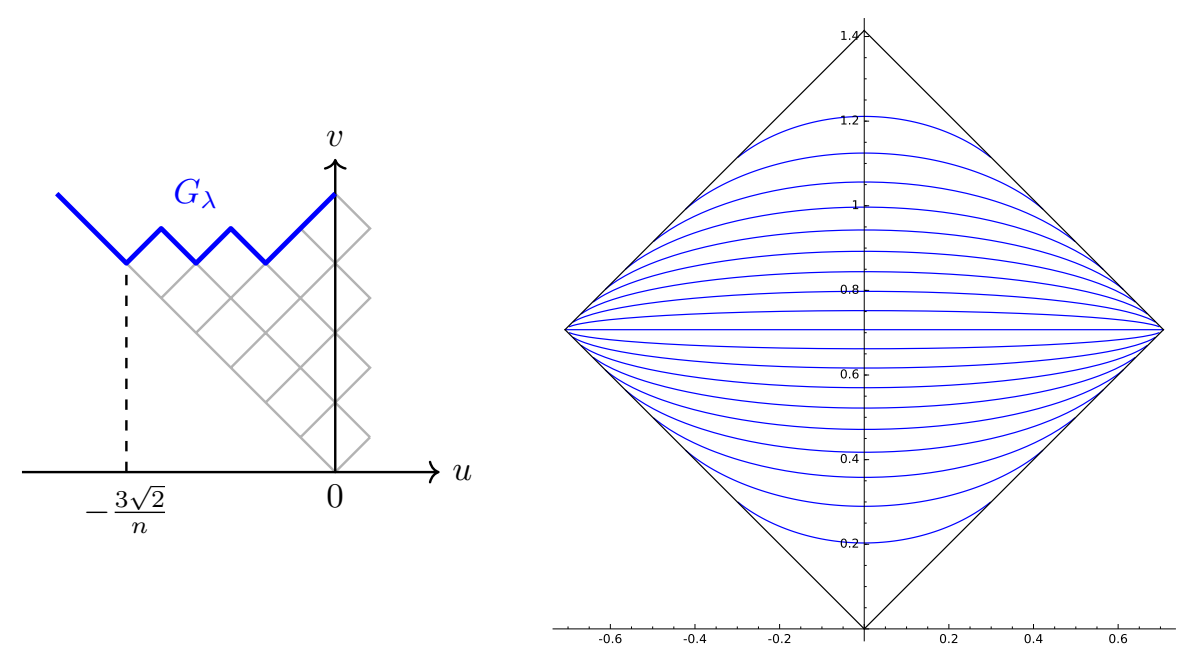

FiguRE 12. Left: the function $G_{\lambda}$ for the partition $(6,4,2,1)$. Right: the curves $v=g_{\alpha}(u)$ for $\alpha=0.05,0.1, \ldots, 0.95$.

LEMMA 3.3. Let $\alpha \in(0,1), k=k(n)$ be a sequence such that $k / N \rightarrow \alpha$ as $n \rightarrow \infty$, and let $\mathbb{P}_{n}$ denote the uniform probability measure on $\mathcal{T}_{n}$. Then, as $n \rightarrow \infty$,

$$
\mathbb{P}_{n}\left(T \in \mathcal{T}_{n}: T\left(\lambda^{\mathrm{sh}}\right)=[k]\right)=\exp \left(-(1+o(1)) \frac{n^{2}}{2}\left(I\left(\gamma_{\Lambda}\right)+H(\alpha)+C\right)\right)
$$


uniformly over all strict partitions $\lambda$ of $k$ with $\lambda_{1}<n$, where

$$
\begin{aligned}
C & =\frac{3}{2}-2 \ln 2, \\
H(\alpha) & =-\alpha \ln (\alpha)-(1-\alpha) \ln (1-\alpha), \\
I(\gamma) & =\int_{0}^{1} \int_{0}^{1} \ln \left|\gamma(x)+\gamma^{-1}(y)-x-y\right| \mathrm{d} y \mathrm{~d} x, \\
\gamma^{-1}(y) & =\inf \{x \in[0,1]: \gamma(x) \leqslant y\},
\end{aligned}
$$

and $\Lambda$ denotes the double of $\lambda$.

Proof. We use Lemma 3.2 and proceed exactly as in the proof of [27, Lem. 1]. Compared to [27] our partitions lie inside an $(n-1) \times n$ rectangle rather than a square, however, the obtained error is small as $n$ tends to infinity. Also note that

$$
-\ln \left(\left(\begin{array}{c}
N \\
k
\end{array}\right)^{-1} \cdot \sqrt{\left(\begin{array}{c}
2 N \\
2 k
\end{array}\right)}\right)=-\frac{1}{4} \ln (\alpha(1-\alpha) \pi N)+\mathcal{O}(1)
$$

as $n \rightarrow \infty$ by Stirling's approximation, thus these terms do not contribute to the analysis.

Results of the type of Lemma 3.3 lead to a so-called large deviation principle. Suppose that $\lambda$ is the strict partition of size $k$ with $\lambda_{1}<n$ such that its double $\Lambda$ minimises the integral $I\left(\gamma_{\Lambda}\right)$. If $\mu$ is a different strict partition of size $k$ with $\mu_{1}<$ $n$ then by Lemma 3.3 the probability that $\mu$ contains the numbers $1, \ldots, k$ in a random shifted SYT $T \in \mathcal{T}_{n}$ decays exponentially as $\mu$ deviates from $\lambda$. This means that the shape formed by the entries $1, \ldots, k$ in a random shifted SYT will be close to the minimising partition $\lambda$ with high probability. One is therefore lead to the variational problem of identifying the function $\gamma$ within a certain search space of functions depending on $\alpha$ that minimises the integral $I(\gamma)$.

A function

$$
g:[-\sqrt{2} / 2, \sqrt{2} / 2] \rightarrow[0, \sqrt{2}]
$$

is called $\alpha$-admissible if it is 1-Lipschitz and satisfies

$$
\int_{-\sqrt{2} / 2}^{\sqrt{2} / 2}(g(u)-|u|) \mathrm{d} u=\alpha .
$$

As is explained in [27, Sec. 2.2] our problem is equivalent to the following formulation: For each $\alpha \in(0,1)$ find the unique $\alpha$-admissible function $g$ which is symmetric, that is, $g(-u)=g(u)$, and minimises the integral

$$
K(g)=-\frac{1}{2} \int_{-\sqrt{2} / 2}^{\sqrt{2} / 2} \int_{-\sqrt{2} / 2}^{\sqrt{2} / 2} g^{\prime}(s) g^{\prime}(t) \ln |s-t| \mathrm{d} s \mathrm{~d} t .
$$

The only difference between our situation and the situation in [27] is the fact that our search space of admissible functions $g$ is smaller since we require that $\Lambda$ is the double of a strict partition. In [27, Sec. 2 and 3] Pittel and Romik show that the variational problem $(8)$ without the assumption $g(-u)=g(u)$ has the unique solution $\tilde{g}_{\alpha}$ given by

$$
\tilde{g}_{\alpha}(u)= \begin{cases}g_{\alpha}(u) & \text { if }|u| \leqslant \sqrt{2 \alpha(1-\alpha)} \\ |u| & \text { if } \sqrt{2 \alpha(1-\alpha)} \leqslant|u| \leqslant \sqrt{2} / 2\end{cases}
$$

where

$$
g_{\alpha}:[-\sqrt{2 \alpha(1-\alpha)}, \sqrt{2 \alpha(1-\alpha)}] \rightarrow \mathbb{R}
$$


is defined as

$$
g_{\alpha}(u)=\frac{2 u}{\pi} \tan ^{-1}\left(\frac{(1-2 \alpha) u}{\sqrt{2 \alpha(1-\alpha)-u^{2}}}\right)+\frac{\sqrt{2}}{\pi} \tan ^{-1}\left(\frac{\sqrt{2\left(2 \alpha(1-\alpha)-u^{2}\right)}}{1-2 \alpha}\right),
$$

if $0<\alpha<1 / 2$, and

for $1 / 2<\alpha<1$, and

$$
\tilde{g}_{\alpha}(u)=\sqrt{2}-\tilde{g}_{1-\alpha}(u)
$$

$$
\tilde{g}_{1 / 2}(u)=\frac{\sqrt{2}}{2} .
$$

The family of functions $g_{\alpha}$ is illustrated in Figure 12. Since this solution already exhibits the additional symmetry $\tilde{g}_{\alpha}(-u)=\tilde{g}_{\alpha}(u)$ as $\tan ^{-1}$ is odd, we may apply it to the shifted case as well.

Let

$$
\bar{L}:\left\{(u, v) \in \mathbb{R}^{2}:-\sqrt{2} / 2 \leqslant u \leqslant 0,|u| \leqslant v \leqslant \sqrt{2}-|u|\right\} \rightarrow \mathbb{R}_{\geqslant 0}
$$

be the surface defined by the level curves $v=g_{\alpha}(u)$ for $\alpha \in(0,1)$. Let

$$
L:\left\{(x, y) \in \mathbb{R}^{2}: 0 \leqslant x \leqslant y \leqslant 1\right\} \rightarrow \mathbb{R}_{\geqslant 0}
$$

be the rotated version of $\bar{L}$, that is, $L(x, y)=\bar{L}(u, v)$, where $(x, y)$ and $(u, v)$ are related as in (7).

The following lemma collects analytic results on the integral $K(g)$ and the functions $\tilde{g}_{\alpha}$ that are found in [27].

LEMMA 3.4.

(i) There exists a constant $c_{K}>0$ such that for all 1-Lipschitz functions $g, h$ we have

$$
|K(g)-K(h)| \leqslant c_{K}\|g-h\|_{\infty} .
$$

(ii) There exists a function $c:(2,3) \rightarrow \mathbb{R}_{>0}$ such that for all $r \in(2,3)$ and all $\alpha$-admissible functions $g$ we have

$$
K(g)+H(\alpha)-\ln 2 \geqslant c(r)\left\|g-\tilde{g}_{\alpha}\right\|_{\infty}^{r} .
$$

(iii) Let $(x, y) \in(0,1) \times(0,1)$, let $(u, v)$ be given as in $(7)$, set $\alpha=\bar{L}(u, v)$, and set

$$
\sigma(x, y)=\min (x y,(1-x)(1-y)) .
$$

Then there exist constants $c_{1}>0$ and $c_{2}>0$ such that for all $\beta \in(0,1)$ and all $\delta<c_{2} \sigma(x, y)^{2}$ we have

$$
\left|\tilde{g}_{\alpha}(u)-\tilde{g}_{\beta}(u)\right|<\delta c_{1} \sqrt{\sigma(x, y)} \quad \Rightarrow \quad|\alpha-\beta|<\delta .
$$

Proof. Claim (i) follows from the proof of [27, Lem. 2]. Claim (ii) is an immediate consequence of $[27$, Thm. 5 and Lem. 4]. Claim (iii) is precisely the statement of [27, Lem. 5].

The last ingredient that is needed to obtain the limit shape for random shifted SYT of staircase shape is a bound on the expected number of entries less than $k$ in the first row of such a tableau. We start with an auxiliary result. Given a partition $\lambda$ let $\lambda^{+}$denote the partition obtained from $\lambda$ by adding a cell to the first row, that is, $\lambda^{+}=\left(\lambda_{1}+1, \lambda_{2}, \ldots, \lambda_{\ell(\lambda)}\right)$.

LEMMA 3.5. Let $m, n \in \mathbb{N}, \square=\left(n^{m}\right)$ be a rectangle and $\lambda$ a partition such that $\left(\lambda^{+}\right)^{\mathrm{dg}} \subseteq \square^{\mathrm{dg}}$. Then

$$
\frac{f_{\lambda} \cdot f_{\square \backslash \lambda^{+}}}{f_{\lambda^{+}} \cdot f_{\square \backslash \lambda}}=\frac{\left(m+\lambda_{1}\right)\left(n-\lambda_{1}\right)}{(|\lambda|+1)(m n-|\lambda|)} .
$$


Proof. This was proved by Pittel and Romik in the case where $\square$ is an $n$ times $n$ square [27, Eq. (71)]. The proof of the generalisation to rectangles relies on the same idea.

First note that

$$
\frac{f_{\lambda}}{f_{\lambda^{+}}}=\frac{|\lambda| !}{(|\lambda|+1) !} \prod_{u \in \lambda^{\mathrm{d} g}} \frac{h_{\lambda^{+}}(u)}{h_{\lambda}(u)}=\frac{1}{(|\lambda|+1)} \prod_{j=1}^{\lambda_{1}} \frac{h_{\lambda^{+}}(1, j)}{h_{\lambda}(1, j)} .
$$

Divide $\left[\lambda_{1}\right]$ into maximal sub-intervals $\left[i_{r}, j_{r}\right]$ such that $\lambda_{j}^{\prime}=\lambda_{i_{r}}^{\prime}$ for all $i_{r} \leqslant j \leqslant j_{r}$. After even more cancellations (10) is equal to

$$
\frac{1}{(|\lambda|+1)} \prod_{r} \frac{h_{\lambda^{+}}\left(1, i_{r}\right)}{h_{\lambda}\left(1, j_{r}\right)}=\frac{1}{(|\lambda|+1)} \prod_{r} \frac{\lambda_{1}-i_{r}+\lambda_{i_{r}}^{\prime}+1}{\lambda_{1}-j_{r}+\lambda_{j_{r}}^{\prime}},
$$

which we rewrite as

$$
\frac{\chi}{(|\lambda|+1)} \prod_{\left(u_{1}, u_{2}\right) \in A}\left(\lambda_{1}-u_{2}+u_{1}\right)^{-1} \prod_{\left(u_{1}, u_{2}\right) \in B}\left(\lambda_{1}-u_{2}+u_{1}\right),
$$

where $A$ denotes the set of cells $u \in \lambda^{\mathrm{dg}}$ such that $\mathbf{e} u, \mathbf{s} u \notin \lambda^{\mathrm{dg}}, B$ denotes the set of cells $u \in \square^{\mathrm{dg}} \backslash \lambda^{\mathrm{dg}}$ such that $\mathbf{n} u, \mathbf{w} u \notin\left(\square^{\mathrm{dg}} \backslash \lambda^{\mathrm{dg}}\right)$, and

$$
\chi= \begin{cases}\left(m+\lambda_{1}\right) & \text { if } \ell(\lambda)=m \\ 1 & \text { otherwise }\end{cases}
$$

Divide the set $[m-1]$ into maximal sub-intervals $\left[i_{r}, j_{r}\right]$ such that $\left(\square \backslash \lambda^{+}\right)_{j}=$ $n-\lambda_{m+1-i_{r}}$ for all $i_{r} \leqslant j \leqslant j_{r}$. Then

$$
\frac{f_{\square \backslash \lambda^{+}}}{f_{\square \backslash \lambda}}=\frac{n-\lambda_{1}}{(m n-|\lambda|)} \prod_{r} \frac{h_{\square \backslash \lambda}\left(n-\lambda_{1}, i_{r}\right)}{h_{\square \backslash \lambda^{+}}\left(n-\lambda_{1}, j_{r}\right)},
$$

which can be written as

$$
\frac{\left(n-\lambda_{1}\right) \cdot \bar{\chi}}{(m n-|\lambda|)} \prod_{\left(u_{1}, u_{2}\right) \in A}\left(\lambda_{1}-u_{2}+u_{1}\right) \prod_{\left(u_{1}, u_{2}\right) \in B}\left(\lambda_{1}-u_{2}+u_{1}\right)^{-1},
$$

where $A$ and $B$ are defined as above, and

$$
\bar{\chi}= \begin{cases}1 & \text { if } \ell(\lambda)=m, \\ \left(m+\lambda_{1}\right) & \text { otherwise. }\end{cases}
$$

The claim now follows from the observation that almost all factors that appear in (11) and (12) cancel.

The following lemma provides us with an analogue of [27, Eq. (75)] for the shifted case. Given $n, k \in \mathbb{N}$ with $k<N$, let $I_{n, k}: \mathcal{T}_{n} \rightarrow\{0,1\}$ denote the random variable that takes the value 1 if the entry $k$ is contained in the first row, and 0 otherwise. Moreover, let $J_{n, k}=\sum_{i=1}^{k} I_{n, i}$ denote the number of entries at most $k$ in the first row of a shifted SYT.

LEMmA 3.6. Let $k, n \in \mathbb{N}$ with $1<k<N$, and let $\mathbb{E}_{n}$ denote the expected value with respect to the uniform probability measure $\mathbb{P}_{n}$ on $\mathcal{T}_{n}$. Then

$$
\left(\mathbb{E}_{n}\left[I_{n, k}\right]\right)^{2}<\mathbb{E}_{n}\left[\frac{2 N-\left(J_{n, k-1}\right)^{2}}{k(N-k+1)}\right]
$$


Proof. Our proof is very similar to the first part of the proof of [27, Lem. 10].

Let $\mathcal{Y}_{n, k}$ denote the set of all strict partitions $\lambda$ of size $k$ with $\lambda_{1}<n-1$. Note that

$$
\begin{aligned}
\mathbb{E}_{n}\left[I_{n, k}\right] & =\mathbb{P}_{n}\left(T \in \mathcal{T}_{n}: T^{-1}(k)=(1, j) \text { for some } j \in[n-1]\right) \\
& =\sum_{\lambda \in \mathcal{Y}_{n, k-1}} \frac{f_{\lambda}^{\mathrm{sh}} \cdot f_{\Delta_{n} \backslash \lambda^{+}}^{\mathrm{sh}}}{f_{\Delta_{n}}^{\mathrm{sh}}} \\
& =\sum_{\lambda \in \mathcal{Y}_{n, k-1}} \frac{f_{\lambda^{+}}^{\mathrm{sh}} \cdot f_{\Delta_{n} \backslash \lambda^{+}}^{\mathrm{sh}}}{f_{\Delta_{n}}^{\mathrm{sh}}} \cdot \frac{f_{\lambda}^{\mathrm{sh}}}{f_{\lambda^{+}}^{\mathrm{sh}}} .
\end{aligned}
$$

Using the fact that $\mu \mapsto f_{\mu}^{\text {sh }} \cdot f_{\Delta_{n} \backslash \mu}^{\text {sh }} / f_{\Delta_{n}}^{\text {sh }}$ defines a probability measure on the set of strict partitions $\mu$ of size $k$ with $\mu_{1}<n$, and the convexity of the square function we obtain

$$
\begin{aligned}
\left(\mathbb{E}_{n}\left[I_{n, k}\right]\right)^{2} & \leqslant \sum_{\lambda \in \mathcal{Y}_{n, k-1}} \frac{f_{\lambda^{+}}^{\mathrm{sh}} \cdot f_{\Delta_{n} \backslash \lambda^{+}}^{\mathrm{sh}}}{f_{\Delta_{n}}^{\mathrm{sh}}} \cdot\left(\frac{f_{\lambda}^{\mathrm{sh}}}{f_{\lambda^{+}}^{\mathrm{sh}}}\right)^{2} \\
& =\sum_{\lambda \in \mathcal{Y}_{n, k-1}} \frac{f_{\lambda}^{\mathrm{sh}} \cdot f_{\Delta_{n} \backslash \lambda}^{\mathrm{sh}}}{f_{\Delta_{n}}^{\mathrm{sh}}} \cdot \frac{f_{\lambda}^{\mathrm{sh}} \cdot f_{\Delta_{n} \backslash \lambda^{+}}^{\mathrm{sh}}}{f_{\lambda^{+}}^{\mathrm{sh}} \cdot f_{\Delta_{n} \backslash \lambda}^{\mathrm{sh}}} .
\end{aligned}
$$

Let $L, M$ and $\square_{n}$ denote the doubles of $\lambda, \lambda^{+}$and $\Delta_{n}$, respectively. Proposition 3.1 yields

$$
\begin{aligned}
\frac{f_{\lambda}^{\mathrm{sh}} \cdot f_{\Delta_{n} \backslash \lambda^{+}}^{\mathrm{sh}}}{f_{\lambda^{+}}^{\text {sh }} \cdot f_{\Delta_{n} \backslash \lambda}^{\text {sh }}} & =4 \cdot \sqrt{\frac{\left(k-\frac{1}{2}\right)\left(N-k+\frac{1}{2}\right)}{k(N-k+1)}} \cdot \sqrt{\frac{f_{L} \cdot f_{\square_{n} \backslash M}}{f_{M} \cdot f_{\square_{n} \backslash L}}} \\
& =4 \cdot \sqrt{\frac{\left(k-\frac{1}{2}\right)\left(N-k+\frac{1}{2}\right)}{k(N-k+1)}} \cdot \sqrt{\frac{f_{L} \cdot f_{\square_{n} \backslash L^{+}}}{f_{L^{+}} \cdot f_{\square_{n} \backslash L}}} \cdot \sqrt{\frac{f_{\left(L^{+}\right)^{\prime}} \cdot f_{\left(\square_{n} \backslash M\right)^{\prime}}}{f_{M^{\prime}} \cdot f_{\left(\square_{n} \backslash L^{+}\right)^{\prime}}}} .
\end{aligned}
$$

Using $M^{\prime}=\left(\left(L^{+}\right)^{\prime}\right)^{+}$and Lemma 3.5 twice we obtain

$$
\frac{f_{L} \cdot f_{\square_{n} \backslash L^{+}}}{f_{L^{+}} \cdot f_{\square_{n} \backslash L}} \cdot \frac{f_{\left(L^{+}\right)^{\prime}} \cdot f_{\left(\square_{n} \backslash M\right)^{\prime}}}{f_{M^{\prime}} \cdot f_{\left(\square_{n} \backslash L^{+}\right)^{\prime}}}=\frac{\left(n+\lambda_{1}\right)^{2}\left(n-\lambda_{1}-1\right)^{2}}{16\left(k-\frac{1}{2}\right)(N-k+1) k\left(N-k+\frac{1}{2}\right)} .
$$

Inserting this into (14) we obtain

$$
\frac{f_{\lambda}^{\mathrm{sh}} \cdot f_{\Delta_{n} \backslash \lambda^{+}}^{\mathrm{sh}}}{f_{\lambda^{+}}^{\mathrm{sh}} \cdot f_{\Delta_{n} \backslash \lambda}^{\mathrm{sh}}}=\frac{\left(n+\lambda_{1}\right)\left(n-\lambda_{1}-1\right)}{k(N-k+1)}<\frac{2 N-\lambda_{1}^{2}}{k(N-k+1)} .
$$

The claim now follows from

$$
\sum_{\lambda \in \mathcal{Y}_{n, k-1}} \frac{f_{\lambda}^{\mathrm{sh}} \cdot f_{\Delta_{n} \backslash \lambda}^{\mathrm{sh}}}{f_{\Delta_{n}}^{\mathrm{sh}}} \cdot \frac{2 N-\lambda_{1}^{2}}{k(N-k+1)}=\mathbb{E}_{n}\left[\frac{2 N-\left(J_{n, k-1}\right)^{2}}{k(N-k+1)}\right] .
$$

To see this note that the first factor in the summand is the probability that the entries $1, \ldots, k-1$ of a random shifted SYT of shape $\Delta_{n}^{\text {sh }}$ are contained precisely in the shifted diagram $\lambda^{\text {sh }}$. Hence the value $\lambda_{1}$ in the summand counts the number of entries less than $k$ that appear in the first row.

We now prove the limit shape theorem for shifted SYT of staircase shape chosen uniformly at random. Our result is an analogue of [27, Thm. 1]. The obtained limit shape is the same as the limit shape for random SYT of square shape except that the domain is restricted from a square to a triangle.

In particular (16) provides point-wise convergence to the limit surface, while (17) specifies the rate of convergence if we assume a sufficient distance to the short sides of the triangle. 
THEOREM 3.7. For $n \in \mathbb{N}$ let $\Delta_{n}$ denote the staircase partition of size $N=\left(\begin{array}{c}n \\ 2\end{array}\right), \mathcal{T}_{n}$ the set of shifted $S Y T$ of shape $\Delta_{n}^{\text {sh }}$, and $\mathbb{P}_{n}$ the uniform probability measure on $\mathcal{T}_{n}$. Then for all $\epsilon>0$

$$
\lim _{n \rightarrow \infty} \mathbb{P}_{n}\left(T \in \mathcal{T}_{n}: \max _{(i, j) \in \Delta_{n}^{\text {sh }}}\left|\frac{T(i, j)}{N}-L\left(\frac{i}{n}, \frac{j}{n}\right)\right|>\epsilon\right)=0 .
$$

Moreover for all $p \in(0,1 / 2)$ and all $q \in(0, p / 2)$ such that $2 p+q<1$

$$
\lim _{n \rightarrow \infty} \mathbb{P}_{n}\left(T \in \mathcal{T}_{n}: \max _{\substack{(i, j) \in \Delta_{n}^{\text {sh }} \\ \sigma(i / n, j / n)>n^{-q}}}\left|\frac{T(i, j)}{N}-L\left(\frac{i}{n}, \frac{j}{n}\right)\right|>n^{-p}\right)=0,
$$

where $\sigma(x, y)=\min \{x y,(1-x)(1-y)\}$.

Proof. The first part (16) is proven in the same way as [27, Thm. 1 (i)] in [27, Sec. 4] with Lemma 3.6 taking the place of [27, Eq. (75)]. The proof of (17) is essentially the same as the proof of $[27$, Thm. 1 (ii)] given in [27, Sec. 2.3] using Lemma 3.3 in place of $[27$, Lem. 1]. Below we only demonstrate the details for (17), for which [27] seems to contain a small inaccuracy.

Let $p^{\prime}(k)$ denote the number of strict partitions of size $k$. Then

$$
p^{\prime}(k) \sim \frac{3^{3 / 4}}{12 k^{3 / 4}} \exp (\pi \sqrt{k / 3})
$$

as $k \rightarrow \infty$. Confer [10, Fig. I.9].

Given $k=\alpha N$ and a tableau $T \in \mathcal{T}_{n}$ let $\lambda_{T, k}$ denote the partition with shifted Young diagram $\lambda_{T, k}^{\mathrm{sh}}=T^{-1}([k])$. Let $\Lambda_{T, k}$ denote the double of $\lambda_{T, k}$. Note that given the double $\Lambda$ of a strict partition $\lambda$ of $k$, the function $g_{\Lambda}$ is 1-Lipschitz but not $\alpha$ admissible, since

$$
\int_{\mathbb{R}} g_{\Lambda}(u)-|u| \mathrm{d} u=\frac{2 \alpha N}{n^{2}}=\alpha\left(1-\frac{1}{n}\right) .
$$

However, we can always choose an $\alpha$-admissible function $\hat{g}_{\Lambda}$ such that

$$
\left\|g_{\Lambda}-\hat{g}_{\Lambda}\right\|_{\infty} \leqslant \frac{\sqrt{2}}{n} .
$$

This is needed below for using Lemma 3.4 (ii). There exists a constant $C>0$ such that for all $r \in(2,3)$ and all $\epsilon_{1}>0$

$$
\begin{aligned}
& \mathbb{P}_{n}\left(T \in \mathcal{T}_{n}:\left\|g_{\Lambda_{T, k}}-\tilde{g}_{\alpha}\right\|_{\infty}>\epsilon_{1}\right) \\
& =\sum_{\substack{\lambda^{\mathrm{sh}} \subseteq \Delta_{n}^{\mathrm{sh}},|\lambda|=k \\
\left\|g_{\Lambda}-\tilde{g}_{\alpha}\right\|_{\infty}>\epsilon_{1}}} \mathbb{P}_{n}\left(T \in \mathcal{T}_{n}: T\left(\lambda^{\mathrm{sh}}\right)=[k]\right) \\
& \stackrel{\text { Lemma }}{3.3} p^{\prime}(k) \max _{\substack{\lambda^{\mathrm{sh}} \subseteq \Delta_{n}^{\mathrm{sh}},|\lambda|=k \\
\left\|g_{\Lambda}-\tilde{g}_{\alpha}\right\|_{\infty}>\epsilon_{1}}} \exp \left(-(1+o(1)) \frac{n^{2}}{2}\left(K\left(g_{\Lambda}\right)+H(\alpha)-\ln 2\right)\right)
\end{aligned}
$$

$$
\stackrel{\substack{(19) \text { and } \\ \text { Lemma } \\=}}{3.4(\mathrm{i})} p^{\prime}(k) \max _{\substack{\lambda^{\mathrm{sh}} \subseteq \Delta_{n}^{\mathrm{sh}},|\lambda|=k \\\left\|g_{\Lambda}-\tilde{g}_{\alpha}\right\|_{\infty}>\epsilon_{1}}} \exp \left(-(1+o(1)) \frac{n^{2}}{2}\left(K\left(\hat{g}_{\Lambda}\right)+H(\alpha)-\ln 2\right)\right)
$$

(18) and

$$
\stackrel{\text { Lemma } 3.4(\mathrm{ii})}{\leqslant} \exp \left(C n-\frac{c(r)}{2} n^{2} \epsilon_{1}^{r}\right)
$$

as $n \rightarrow \infty$

For $(i, j) \in \Delta_{n}^{\mathrm{sh}}$ set $\beta=L(i / n, j / n)$. Note that if $(u, v)$ corresponds to $(x, y)=$ $(i / n, j / n)$, then $\tilde{g}_{\beta}(u)=v$, and since $T\left(\lambda_{T, T(i, j)}\right)=[T(i, j)], G_{\lambda_{T, T(i, j)}}(u)=v$ by the 
definition of $G$. Given $T \in \mathcal{T}_{n}$ set $\alpha_{T}=T(i, j) / N$. For all $r \in(2,3)$ and all $\delta>0$ that satisfy

if $n$ is large enough, then

$$
\delta<c_{2} \sigma(i / n, j / n)^{2}
$$

$$
\begin{aligned}
\mathbb{P}_{n}\left(T \in \mathcal{T}_{n}:\left|\frac{T(i, j)}{N}-L\left(\frac{i}{n}, \frac{j}{n}\right)\right|>\delta\right) \\
\quad=\mathbb{P}_{n}\left(T \in \mathcal{T}_{n}:\left|\alpha_{T}-\beta\right|>\delta\right) \\
\stackrel{\text { Lemma } 3.4(\mathrm{iii)})}{\leqslant} \mathbb{P}_{n}\left(T \in \mathcal{T}_{n}:\left|\tilde{g}_{\alpha_{T}}(u)-\tilde{g}_{\beta}(u)\right|>\delta c_{1} \sqrt{\sigma(i / n, j / n)}\right) \\
=\mathbb{P}_{n}\left(T \in \mathcal{T}_{n}:\left|G_{\lambda_{T, T(i, j)}}(u)-\tilde{g}_{\alpha_{T}}(u)\right|>\delta c_{1} \sqrt{\sigma(i / n, j / n)}\right) \\
\quad \leqslant \mathbb{P}_{n}\left(T \in \mathcal{T}_{n}:\left|g_{\Lambda_{T, T(i, j)}}(u)-\tilde{g}_{\alpha_{T}}(u)\right|>\delta c_{1} \sqrt{\sigma(i / n, j / n)}-\frac{\sqrt{2}}{n}\right) \\
\quad \leqslant \mathbb{P}_{n}\left(T \in \mathcal{T}_{n}:\left\|g_{\Lambda_{T, T(i, j)}}-\tilde{g}_{\alpha_{T}}\right\|_{\infty}>\frac{\delta c_{1}}{2} \sqrt{\sigma(i / n, j / n)}\right) \\
\stackrel{(20)}{\leqslant} \exp \left(C n-\frac{c(r)}{2}\left(\frac{c_{1}}{2}\right)^{r} n^{2}(\delta \sqrt{\sigma(i / n, j / n)})\right.
\end{aligned}
$$

Suppose $\delta=n^{-p}$ and $\sigma(i / n, j / n)>n^{-q}$ for some $p>0$ and $q>0$ such that $p>2 q$ and

Then for $n$ large enough

$$
p+\frac{q}{2}<\frac{1}{2}-\epsilon_{2}
$$

$$
\delta=n^{-p}<n^{-2 q}<c_{2} \sigma(i / n, j / n)^{2}
$$

and

$$
\frac{\delta c_{1}}{2} \sqrt{\sigma(i / n, j / n)}>\frac{c_{1}}{2} n^{-p-q / 2}>n^{-1 / 2+\epsilon_{2}} .
$$

For all $\epsilon_{2}>0$ there exists $\epsilon_{3}>0$ such that the choice

$$
\epsilon_{1}>n^{-1 / 2+\epsilon_{2}}, \quad r=2+\epsilon_{3}
$$

yields

$$
\lim _{n \rightarrow \infty} \exp \left(C n-\frac{c(r)}{2} n^{2} \epsilon_{1}^{r}\right)=0 .
$$

Thus since the number of cells in $\Delta_{n}^{\text {sh }}$ is only quadratic in $n$, we obtain (17) by taking the union bound in (21) over all possible cells.

\section{INTERMEDiATE PERMUTATIONS}

This section contains the derivation of a limit shape of intermediate permutation matrices in a random 132-avoiding sorting network, a parallel to [4, Thm. 2] (previously [1, Conj. 2]).

The (Rothe) diagram of a permutation $\sigma$ is the set

$$
D(\sigma)=\left\{(i, j) \in \mathbb{N}^{2}: i \in\left[i^{\prime}\right] \text { and } j \in\left[j^{\prime}\right] \text { for all } i^{\prime}, j^{\prime} \text { with } M(\sigma)_{i^{\prime}, j^{\prime}}=1\right\}
$$

of cells left unshaded when we shade all the cells weakly to the east and south of 1-entries in the permutation matrix $M(\sigma)$. Recall the definition of $Q_{w}$ above Theorem 2.2 .

TheOrem 4.1 ([20, Thm 3.1, Cor. 3.4]). Let $w=w_{1} \cdots w_{N}$ be a 132-avoiding sorting network and $k \in[N]$. Then $Q_{w_{1} \ldots w_{k}}$ is of shape $D\left(\sigma_{k}\right)$, where $\sigma_{k}=s_{w_{1}} \cdots s_{w_{k}}$. 
Recall the definition of the extension $\tilde{g}_{\alpha}$ of $g_{\alpha}$ to the full interval $[-\sqrt{2} / 2, \sqrt{2} / 2]$ in (9). See also Figure 13a. The theorem below uses Theorem 4.1 to describe the limit shape of intermediate permutation matrices in terms of the extended level curves $\tilde{g}_{\alpha}$. Since we use diagrams of permutations, we have to do this in two parts - first for the indices $j \in[n]$ for which $\sigma_{\lfloor\alpha N\rfloor}(j) \leqslant \sigma_{\lfloor\alpha N\rfloor}(1)$, that is, the columns of $M\left(\sigma_{\lfloor\alpha N\rfloor}\right)$ in which the 1-entry is weakly above the 1-entry in column 1 . The set containing these indices is denoted by $J_{w}(\alpha)$. Secondly, the limit shape can be extended for $j \in[n] \backslash J_{w}(\alpha)$ using a symmetry argument.

ThEOREM 4.2. Let $\sigma_{0}=i d$ and $\sigma_{k}=s_{w_{1}} \cdots s_{w_{k}}$ for $k \in[N]$, where $w=w_{1} \ldots w_{N}$ is a sorting network. Let $\mathbb{P}_{n}$ be the uniform probability measure on $\mathcal{R}_{n}^{132}$, the set of n-element 132-avoiding sorting networks. Finally, let

$$
J_{w}(\alpha)=\left\{j \in[n]: \sigma_{\lfloor\alpha N\rfloor}(j) \leqslant \sigma_{\lfloor\alpha N\rfloor}(1)\right\}
$$

and $J_{w}^{c}(\alpha)=[n] \backslash J_{w}(\alpha)$. For all $0 \leqslant \alpha \leqslant 1$ and $\epsilon>0$,

$$
\mathbb{P}_{n}\left(w \in \mathcal{R}_{n}^{132}: \max _{j \in J_{w}(\alpha)}\left|\frac{\sigma_{\lfloor\alpha N\rfloor}(j)}{n}-\frac{1}{\sqrt{2}}\left(\tilde{g}_{\alpha}\left(\frac{-j}{n \sqrt{2}}\right)-\frac{j}{n \sqrt{2}}\right)\right|>\epsilon\right) \rightarrow 0,
$$

as $n \rightarrow \infty$. By symmetry, for all $0 \leqslant \alpha \leqslant 1$ and $\epsilon>0$,

$$
\mathbb{P}_{n}\left(w \in \mathcal{R}_{n}^{132}: \max _{j \in J_{w}^{c}(\alpha)}\left|\frac{\sigma_{\lfloor\alpha N\rfloor}(j)}{n}+\frac{1}{\sqrt{2}}\left(\tilde{g}_{1-\alpha}\left(\frac{-j}{n \sqrt{2}}\right)-\frac{j}{n \sqrt{2}}\right)-1\right|>\epsilon\right) \rightarrow 0,
$$

as $n \rightarrow \infty$.

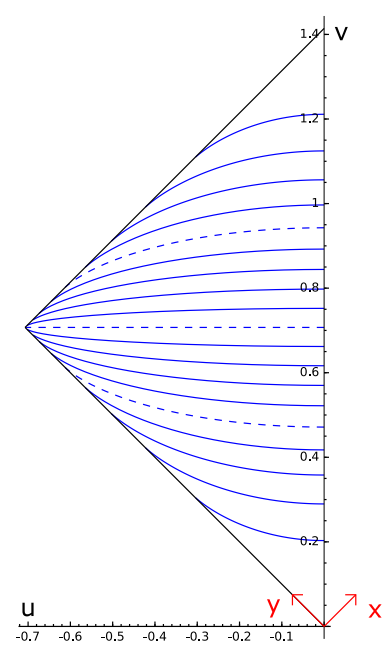

(a) The extended level curves $v=\tilde{g}_{\alpha}(u)$ for $\alpha=0.05,0.1, \ldots, 0.95$.

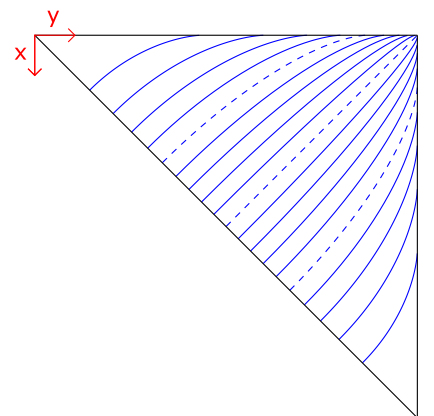

(b) The curves $(x+y) / \sqrt{2}=$ $\tilde{g}_{\alpha}((x-y) / \sqrt{2})$ (the previous picture after rotation).

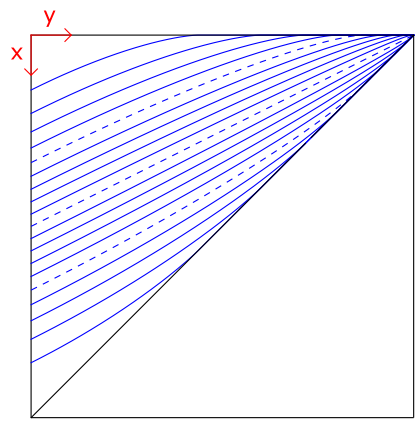

(c) The curves $(2 x+y) / \sqrt{2}=$ $\tilde{g}_{\alpha}(-y / \sqrt{2})$ (the previous picture after shifting to the left).

FiguRE 13. Translating the limit shape of shifted staircase SYT into the limit of the diagrams of the intermediate permutations. The blue curves in (c) are the limit curves of the diagrams of the intermediate permutations at times $\alpha=0.05,0.1, \ldots, 0.95$. The dashed curves correspond to the values $\alpha=0.25,0.5$ and 0.75 , and also appear in Figure 14. 

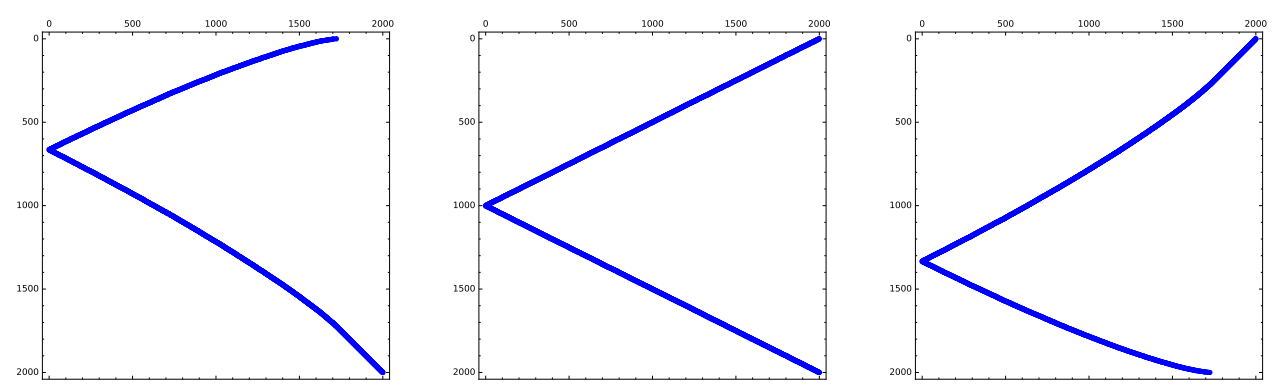

FiguRE 14. The intermediate permutation matrices of a random 132-avoiding sorting network with 2000 elements at times $\alpha=\frac{1}{4}, \frac{1}{2}$ and $\frac{3}{4}$. Compare with Figure $13 \mathrm{c}$ which contains the upper parts of the blue curves (dashed), and also with the general sorting network case in Figure 4.

Proof. By Theorem 3.7 and Theorem 4.1, the diagram of the intermediate permutation matrix at time $\alpha$ scaled by $1 / n$ converges in probability to a (un)shifted version of the (rotated) level curve $\frac{x+y}{\sqrt{2}}=\tilde{g}_{\alpha}\left(\frac{x-y}{\sqrt{2}}\right)$ (Figure 13b). The shift can be performed by sending $y$ to $y+x$ since $y_{\text {shifted }}=y_{\text {non-shifted }}+x$. Thus we get the curve $\frac{2 x+y}{\sqrt{2}}=\tilde{g}_{\alpha}\left(\frac{-y}{\sqrt{2}}\right)$ and consequently the explicit limit formula $x=\frac{1}{\sqrt{2}}\left(\tilde{g}_{\alpha}\left(\frac{-y}{\sqrt{2}}\right)-\frac{y}{\sqrt{2}}\right)$ for $x=\sigma_{\lfloor\alpha N\rfloor}(\lfloor y n\rfloor) / n$. See Figure 13c. The missing part of the limiting permutation matrix is obtained from the diagram by the symmetry in Proposition 2.3.

In particular, at $\alpha=\frac{1}{2}$ the diagram is bounded by the line $y=1-2 x$, which can be seen in Figure 14. Note that $(x, y)=0$ is in the top-left corner.

\section{Trajectories}

Next, inspired by the former sine trajectories conjecture [1, Conj. 1] of Angel et al. (now [4, Thm. 1]), we study trajectories in random 132-avoiding sorting networks.

The trajectory of the element $i \in[n]$ in $w=w_{1} \ldots w_{N}$ is the function $k \mapsto \sigma_{k}^{-1}(i)$. See Figure 15.

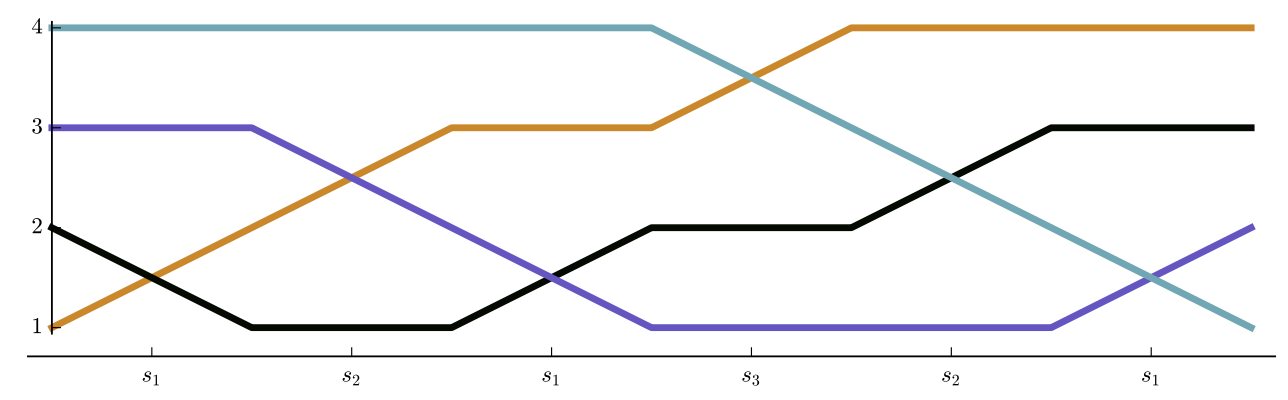

FiguRE 15. Trajectories in the 132-avoiding sorting network 121321. The permutations $\sigma_{k}$ are $\sigma_{0}=1234, \sigma_{1}=2134, \sigma_{2}=2314, \sigma_{3}=$ $3214, \sigma_{4}=3241, \sigma_{5}=3421$ and $\sigma_{6}=4321$. Hence the trajectory of the element 3 is $(3,3,2,1,1,1,2)$.

The scaled trajectory $f_{i}(\alpha)=f_{w, i}(\alpha)$ of $i$ in an $n$-element 132-avoiding sorting network $w$ is defined by

$$
f_{i}(\alpha)=\frac{\sigma_{\alpha N}^{-1}(i)}{n}
$$


for $\alpha N \in \mathbb{Z}$, and by linear interpolation for other $\alpha \in[0,1]$. Figure 16 contains some examples.

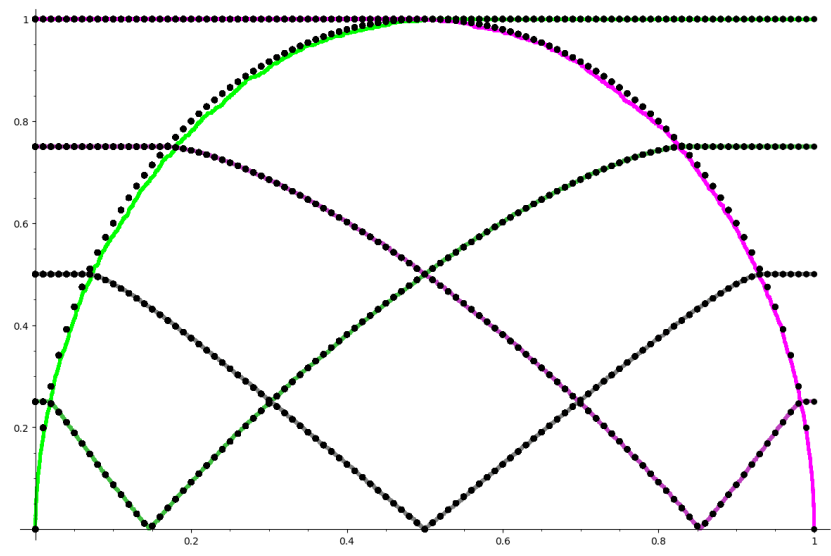

FIgURE 16. The scaled trajectories of the elements 1, 250, 500, 750 and 1000 in a random 132-avoiding sorting network with 1000 elements together with points (in black) from their limits in Theorem 5.4. Compare with Figure 5.

The heights at which different trajectories intersect are deterministic.

PROPOSITION 5.1. In any 132-avoiding sorting network, $i$ and $j$ with $i<j$ are interchanged by $s_{j-i}$.

Proof. We show that all elements between $i$ and $j$ have to pass $i$, and that all elements smaller than $i$ have to pass both $i$ and $j$ before $i$ and $j$ can be swapped. Note that elements $k$ such that $i<k<j$ cannot be swapped with $j$ before they have been swapped with $i$. Otherwise an intermediate permutation would have the pattern 132 . Similarly, all $k^{\prime}$ with $k^{\prime}<i$ have to pass $i$ and hence also $j$ before $i$ and $j$ can be swapped.

In general the element $k$ starts to move when 1 reaches position $k$, that is, at the smallest $t$ such that the first row of the tableau $Q_{w_{1} \ldots w_{t}}$ (defined above Theorem 2.2) has length $k-1$. Figure 16 shows how the trajectories are constant until they are intersected by the trajectory of 1 . This time is given by the limit shape of shifted SYT of staircase shape, and is equal to $\left(1-\sqrt{1-x^{2}}\right) / 2$, where $x=k / n$. This is the content of the proposition below.

Proposition 5.2. For $n \in \mathbb{N}$ let $\mathcal{R}_{n}^{132}$ denote the set of $n$-element 132-avoiding sorting networks and $\mathbb{P}_{n}$ be the uniform probability measure on $\mathcal{R}_{n}^{132}$. Then for all $\epsilon>0$,

$$
\lim _{n \rightarrow \infty} \mathbb{P}_{n}\left(w \in \mathcal{R}_{n}^{132}: \sup _{0 \leqslant \alpha \leqslant 1}\left|f_{w, 1}(\alpha)-\mathfrak{t}_{0}(\alpha)\right|>\epsilon\right)=0,
$$

where

$$
\mathfrak{t}_{0}(\alpha)= \begin{cases}2 \sqrt{\alpha-\alpha^{2}} & \text { if } 0 \leqslant \alpha \leqslant \frac{1}{2}, \\ 1 & \text { if } \frac{1}{2}<\alpha \leqslant 1 .\end{cases}
$$

Proof. By the Edelman-Greene bijection, $f_{w, 1}(\alpha)$ is the length of the first row of $Q_{w_{1} \ldots w_{\lfloor\alpha N\rfloor}}$, scaled by $1 / n$. Let $0 \leqslant \alpha \leqslant \frac{1}{2}$. By Theorem 3.7 and using that

$$
L\left(0, \frac{j}{n}\right)=\frac{1-\sqrt{1-(j / n)^{2}}}{2}
$$


by the definition of $L$ via its level curves $v=g_{\beta}(u)$, for every $\epsilon>0$

$$
\lim _{n \rightarrow \infty} \mathbb{P}_{n}\left(T \in \mathcal{T}_{n}: \max _{j \in[n-1]}\left|\frac{T(1, j)}{N}-\frac{1-\sqrt{1-(j / n)^{2}}}{2}\right|>\epsilon\right)=0 .
$$

The function

$$
\frac{1-\sqrt{1-x^{2}}}{2}
$$

is continuous and strictly increasing. Hence its inverse is also continuous, so for every $\epsilon>0$ there exists a $\delta>0$ such that

$$
\left|\frac{T(1, j)}{N}-\frac{1-\sqrt{1-(j / n)^{2}}}{2}\right|<\delta \Rightarrow\left|\frac{j}{n}-2 \sqrt{\frac{T(1, j)}{N}-\left(\frac{T(1, j)}{N}\right)^{2}}\right|<\epsilon .
$$

This proves the claim with $f_{w, 1}(\alpha)=j / n$ and $\alpha=T(1, j) / N$. Note that since $2 \sqrt{\alpha-\alpha^{2}}=1$ at $\alpha=\frac{1}{2}, \mathfrak{t}_{0}(\alpha)$ has to equal 1 for $\frac{1}{2}<\alpha \leqslant 1$.

Note that by symmetry, the limit shape of the trajectory of $n$ can be obtained by the transformation $\alpha \mapsto 1-\alpha$.

In general, the limit shapes of trajectories are determined by the limit shape of shifted SYT of staircase shape. The lemma below states that we can read the steps at which the element $m$ switches position - and hence the position of $m$ at a given step - by reading the entries of $Q_{w}$ first along the anti-diagonal $i+j-1=m-1$ and then along the column $i=m$.

LEMma 5.3. The trajectory of an element $m \in[n]$ in any n-element 132-avoiding sorting network $w$ is determined by $Q_{w}$ along the anti-diagonal $i+j-1=m-1$ and the row $i=m$. Namely, let

$$
D_{m}=\left\{(i, j) \in \Delta_{n}: i+j-1=m-1 \text { or } i=m\right\} .
$$

Then

$$
\sigma_{k}^{-1}(m)= \begin{cases}m, & \text { if } k<Q_{w}(1, m), \\ \operatorname{pr}_{2}\left(Q_{w}^{-1}\left(\max \left\{k^{\prime} \leqslant k: Q_{w}^{-1}\left(k^{\prime}\right) \in D_{m}\right\}\right)\right), & \text { otherwise, }\end{cases}
$$

where $\operatorname{pr}_{2}(i, j)=j$.

Proof. This follows from Theorem 4.1 by the observation that the element $m$ can only switch places with elements $m^{\prime}<m$ until $m$ reaches the first column, and that $m^{\prime}+1$ cannot pass $m$ before $m^{\prime}$ has, that is, the row $m^{\prime}$ has reached length $m-m^{\prime}$. This proves the part concerning the anti-diagonal $i+j-1=m-1$. The remaining part follows by the symmetry in Proposition 2.3.

The next theorem formulates Lemma 5.3 in the limit in terms of the limit shape in Theorem 3.7. The function $\mathfrak{f}_{\beta}(\alpha)$ below is the non-constant part of the limit of the trajectory of $\lfloor\beta n\rfloor$ and is determined by the limit surface.

TheOREM 5.4. Fix $m / n=\beta$. Let

$$
D_{\beta}=\left\{(x, y) \in \mathbb{R}^{2}: 0 \leqslant x \leqslant y \leqslant 1, y=\beta \text { or } x=\beta\right\} .
$$

Define $\mathfrak{f}_{\beta}(\alpha)=y-x$, where $L^{-1}(\alpha)=(x, y) \in D_{\beta}$, and

$$
\mathfrak{t}_{\beta}(\alpha)= \begin{cases}\beta & \text { if } 0 \leqslant \alpha \leqslant \frac{1-\sqrt{1-\beta^{2}}}{2} \\ \mathfrak{f}_{\beta}(\alpha) & \text { if } \frac{1-\sqrt{1-\beta^{2}}}{2}<\alpha<\frac{1+\sqrt{2 \beta-\beta^{2}}}{2} \\ 1-\beta & \text { if } \frac{1+\sqrt{2 \beta-\beta^{2}}}{2} \leqslant \alpha \leqslant 1\end{cases}
$$


Finally, let $\mathcal{R}_{n}^{132}$ denote the set of $n$-element 132-avoiding sorting networks and let $\mathbb{P}_{n}$ be the uniform probability measure on $\mathcal{R}_{n}^{132}$. Then for all $\epsilon>0$,

$$
\lim _{n \rightarrow \infty} \mathbb{P}_{n}\left(w \in \mathcal{R}_{n}^{132}: \sup _{0 \leqslant \alpha \leqslant 1}\left|f_{w,\lfloor\beta n\rfloor}(\alpha)-\mathfrak{t}_{\beta}(\alpha)\right|>\epsilon\right)=0 .
$$

Proof. This is simply a shifted and scaled version of Lemma 5.3 together with Theorem 3.7. Note that $L(x, y)$ restricted to $D_{\beta}$ is continuous by the continuity of $L(x, y)$ and also strictly increasing since $D_{\beta}$ intersects each level curve exactly once.

Informally, by the result above we can trace the trajectory of $\lfloor\beta n\rfloor$ by following the limit shape along $y=\beta$ until $x=y$, and then along $x=\beta$. If the height $\alpha=L\left(x^{\prime}, y^{\prime}\right)$ is given by some point $\left(x^{\prime}, y^{\prime}\right)$ along this curve, then the trajectory of $\lfloor\beta n\rfloor$ is at height $y^{\prime}-x^{\prime}$ at time $\alpha$. Figure 17 illustrates this. Combined with the implicit definition of $L(x, y)$, this means that it is difficult to compute the trajectories of arbitrary elements explicitly. However, certain values are obtainable for all of the trajectories. In the limit, with high probability, the trajectory of $\lfloor\beta n\rfloor$ is at height 1 at

$$
\alpha= \begin{cases}\frac{1}{2}-\frac{1}{2 \sqrt{1+\tan ^{2}(\pi \beta)}} & \text { if } 0 \leqslant \beta<\frac{1}{2}, \\ \frac{1}{2} & \text { if } \beta=\frac{1}{2}, \\ \frac{1}{2}+\frac{1}{2 \sqrt{1+\tan ^{2}(\pi \beta)}} & \text { if } \frac{1}{2}<\beta \leqslant 1 .\end{cases}
$$

Note that by the proof of Lemma 5.3, this is the time $\alpha$ at which the diagonal side of $Q_{w_{1} \ldots w_{\lfloor\alpha N\rfloor}}$ has reached length $\lfloor\beta n\rfloor$. On the other hand, at time $\alpha=\frac{1}{2}$, the trajectory of $\lfloor\beta n\rfloor$ is at height $\lfloor(1-2 \beta) n\rfloor$ with high probability. Points from some of the scaled limit trajectories predicted by Theorem 5.4 are plotted in Figure 16 together with the corresponding sampled scaled trajectories.
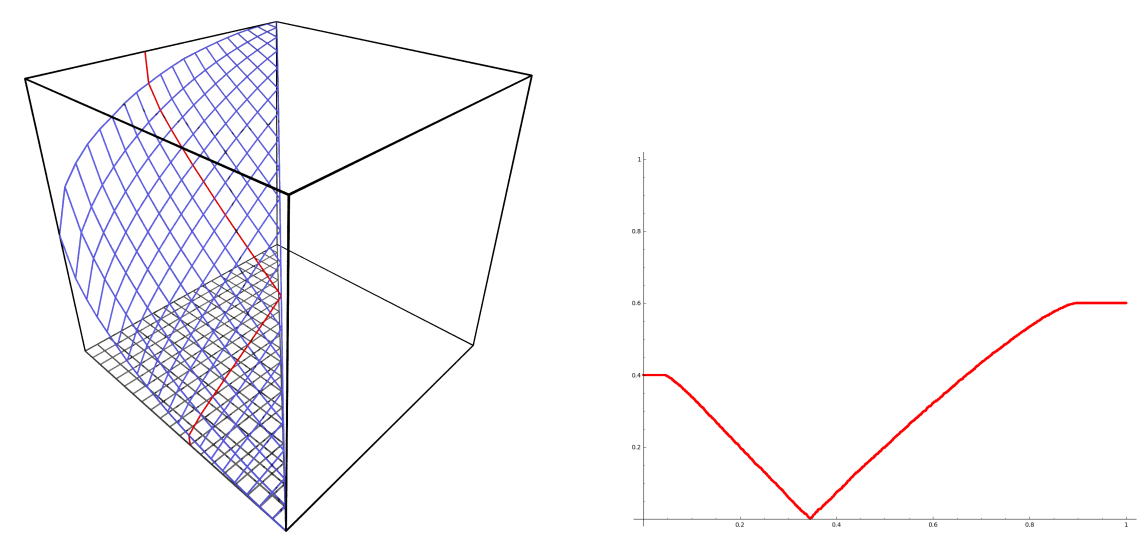

FiguRE 17. A curve (in red) determining the trajectory of $\lfloor\beta n\rfloor$, $\beta=0.4$, shown on the right from the limit shape.

\section{AdJACENCIES}

Motivated by the former great circle conjecture [1, Conj. 3] (now [4, Thm. 4]) and trying to understand the geometry of random 132-avoiding sorting networks on the permutahedron, we next study adjacencies. Note that by $[1$, Thm. 5$]$ and the previous 
two sections, random 132-avoiding sorting networks are not likely to be close to any fixed great circle.

Let $w$ be a reduced word of the longest element $w_{0}$ in $\mathfrak{S}_{n}$. An index $k \in[N-1]$ is called an adjacency of $w$ if $\left|w_{k+1}-w_{k}\right|=1$. Geometrically, adjacencies are special in the following sense.

Proposition 6.1. In the permutahedron, an adjacency $k$ in a reduced word $w$ corresponds to a pair of edges at a $\frac{\pi}{3}$ angle. Otherwise the edges corresponding to consecutive letters $w_{k}$ and $w_{k+1}$ are orthogonal.

Proof. An adjacency corresponds to a pair of adjacent edges of length $\sqrt{2}$ with direction vectors of the form $(0, \ldots, 0,1,0, \ldots, 0,-1,0, \ldots, 0)$ where the indices of either the $1 \mathrm{~s}$ or the $-1 \mathrm{~s}$ coincide. Hence their scalar product is 1 and the edges constitute an angle of $\frac{\pi}{3}$. In the case of $\left|w_{k+1}-w_{k}\right|>1$ the edges corresponding to $w_{k}$ and $w_{k+1}$ are orthogonal.

Adjacencies in a 132-avoiding sorting network $w$ correspond directly to adjacencies in the SYT $Q_{w} \rightarrow$ defined above Theorem 2.2 as follows. Let $\lambda^{\text {dg }}$ be a Young diagram. A pair $(T, u)$ of a cell $u \in \lambda^{\mathrm{dg}}$ and a SYT $T$ of shape $\lambda^{\mathrm{dg}}$ is called a horizontal adjacency if $T(\mathbf{e} u)=T(u)+1$. The pair $(T, u)$ is called a vertical adjacency if $T(\mathbf{s} u)=T(u)+1$. An adjacency $(T, u)$ is said to lie in column $j$ of $\lambda^{\mathrm{dg}}$ if $u=(i, j)$. Likewise, in such a case $(T, u)$ is said to lie in row $i$ of $\lambda^{\mathrm{dg}}$. The definitions are the same for shifted diagrams $\lambda^{\mathrm{sh}}$. For example, consider the tableau $T$ in Figure 18. Then $(T,(1,1))$, $(T,(1,2))$ and $(T,(2,2))$ are horizontal adjacencies whereas $(T,(2,3))$ is a vertical adjacency. The adjacencies lie in columns 1, 2, 2, and 3 (and rows 1, 1, 2 and 2), respectively.
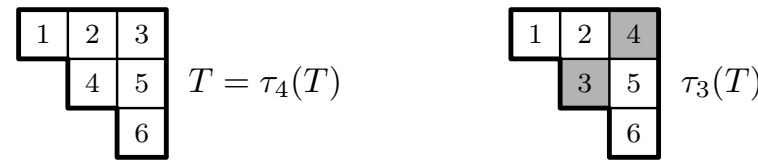

Figure 18. The three shifted SYT $T, \tau_{3}(T)$ and $\tau_{4}(T)$.

Proposition 6.2. Let $w$ be a 132-avoiding sorting network. Then $w_{k+1}=w_{k}+1$ if and only if $\left(Q_{w},\left(Q_{w}\right)^{-1}(k)\right)$ is a horizontal adjacency. Similarly $w_{k+1}=w_{k}-1$ if and only if $\left(Q_{w}^{\vec{w}},\left(Q_{w}\right)^{-1}(k)\right)$ is a vertical adjacency.

Proof. This follows from the fact that $w_{i}$ is inserted in column $w_{i}$ of $Q_{w_{1} \ldots w_{i-1}}$ in the Edelman-Greene bijection, and that $Q_{w}$ can be shifted.

Our next goal is to enumerate adjacencies in SYT. To this end define

$$
\tau_{k}(T)= \begin{cases}T & \text { if }\left(T, T^{-1}(k)\right) \text { is an adjacency, } \\ s_{k} \circ T & \text { otherwise. }\end{cases}
$$

In other words, $\tau_{k}(T)$ exchanges the positions of $k$ and $k+1$ if possible, that is, unless they are adjacent in $T$. See Figure 18.

We consider the bijection $\partial_{k}=\tau_{|\lambda|-1} \circ \cdots \circ \tau_{k+1} \circ \tau_{k}$ from the set of (possibly shifted) SYT of shape $\lambda$ to itself, where $|\lambda|$ is the largest entry in an SYT of shape $\lambda$. Applying $\partial_{k}$ to $T$ can be described by the following procedure called partial promotion:

(1) Form the sequence of cells $u_{0}, \ldots, u_{m}$, called the promotion path, such that

- $u_{0}=T^{-1}(k)$,

- $u_{l+1}=\arg \min \left\{T\left(\mathbf{e} u_{l}\right), T\left(\mathbf{s} u_{l}\right)\right\}$. If at some $l=m$ both are undefined, we stop. 
(2) Remove the label of $u_{0}$ and slide the entries $T\left(u_{0}\right) \leftarrow T\left(u_{1}\right) \leftarrow \cdots \leftarrow T\left(u_{m}\right)$.

(3) Subtract 1 from each label at least $k+1$ and insert $|\lambda|$ into $u_{m}$.

The inverse of $\partial_{k}$ can be described in a similar way:

(1) Form the sequence of cells $u_{m}, \ldots, u_{0}$, called the inverse promotion path, such that

- $u_{m}=T^{-1}(|\lambda|)$

- $u_{l+1}=\arg \max \left\{T\left(\mathbf{w} u_{l}\right), T\left(\mathbf{n} u_{l}\right)\right\}$,

- and $u_{0}$ is the last possible cell in the sequence such that $T\left(u_{0}\right) \geqslant k$.

(2) Remove the label of $u_{m}$ and slide the entries $T\left(u_{0}\right) \rightarrow \cdots \rightarrow T\left(u_{m}\right)$.

(3) Add 1 to each label at least $k$. Insert $k$ into $u_{0}$.

See Figure 19 for an example. In the case $k=1$ partial promotion becomes Schützenberger's promotion [37]. See also [39]. Let $A_{c}(T)$ be the set of horizontal adjacencies $(T,(i, c))$ in column $c$ of a (possibly shifted) standard Young tableau $T$. Let $\lambda$ be a (possibly shifted) shape and let $A_{c}(\lambda)=\cup_{T} A_{c}(T)$ where $T$ runs over all SYT of shape $\lambda$.
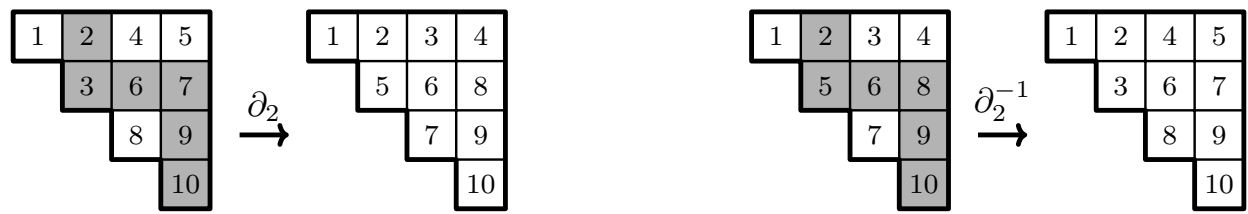

FIGURE 19. Partial promotion $\partial_{2}$ and its inverse, with the promotion paths highlighted.

THEOREM 6.3. For any column $c$ of a (possibly shifted) shape $\lambda$, partial promotion gives a bijection between $A_{c}(\lambda)$ and the set of SYT of shape $\lambda$ with largest entry in or after column $c+1$. The corresponding result for vertical adjacencies is obtained by replacing "column" with "row".

Proof. Consider a tableau $T$ of shape $\lambda$ and assume the largest entry $|\lambda|$ is in column $c+1$ or greater. Then the inverse promotion path of $\tau_{1} \circ \tau_{2} \circ \cdots \circ \tau_{|\lambda|-1}$ has to cross from column $c+1$ to $c$ at some unique $k(T)$, that is, $k(T)$ is the first index at which the path of $\partial_{k(T)}^{-1}=\tau_{k(T)} \circ \cdots \circ \tau_{|\lambda|-1}$ ends in column $c$ of $T$. By the definition of inverse partial promotion, the choice of $k(T)$ ensures that $k(T)$ and $k(T)+1$ are horizontally adjacent with $k(T)$ in some cell $u$ in column $c$ of $\partial_{k(T)}^{-1}(T)$. This is illustrated in Figure 20. Hence, by letting $\phi(T)=\left(\partial_{k(T)}^{-1}(T), u\right)$ we obtain a map $\phi: S_{c}(\lambda) \rightarrow A_{c}(\lambda)$.

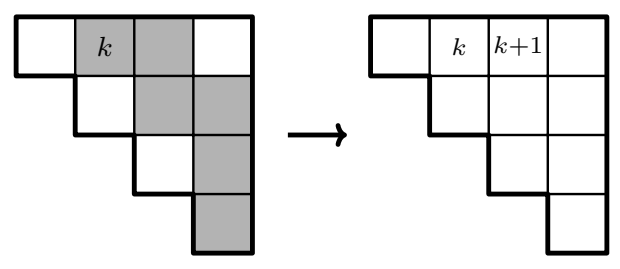

Figure 20. The bijection in the proof of Theorem 6.3 with $k=k(T)$ and the path of $\tau_{k(T)} \circ \cdots \circ \tau_{|\lambda|-1}$ coloured.

If $(S, u)$ is an adjacency in column $c$, the largest entry of $\partial_{S(u)}(S)$ has to be in column $c+1$ or greater as there is at least one entry in column $c+1$. Hence, by defining $\psi(S, u)=\partial_{S(u)}(S)$ we obtain a map $\psi: A_{c}(\lambda) \rightarrow S_{c}(\lambda)$. 
Given the adjacency $\left(\partial_{k(T)}^{-1}(T), u\right)$ in column $c$, note that $\partial_{k(T)}^{-1}(T)(u)=k(T)$, so $\psi(\phi(T))=\partial_{k(T)}\left(\partial_{k(T)}^{-1}(T)\right)=T$. On the other hand, if $(S, u)$ is an adjacency in column $c$, then $\partial_{S(u)}^{-1}\left(\partial_{S(u)}(S)\right)=S$ so $k\left(\partial_{S(u)}(S)\right)=S(u)$. Hence, $\phi(\psi(S, u))=\phi\left(\partial_{S(u)}(S)\right)=\left(\partial_{S(u)}^{-1}\left(\partial_{S(u)}(S)\right), u\right)=(S, u)$. Thus $\phi$ and $\psi$ are inverse bijections and the proof is complete.

The proof works for vertical adjacencies as well if one replaces "column" by "row" since promotion and its inverse are symmetric in rows and columns.

Techniques similar to those used in the proof of Theorem 6.3 also appear in [36], for example in the proof of Theorem 1.3 in [36, Section 3]. Theorem 6.3 is true in a greater generality, for example for many skew shapes. The important feature is that every possible promotion path from the maximal entry has to cross column $c$.

A (shifted) Young diagram is called a (shifted) rectangle if all standard tableaux of its shape contain the largest entry in the same cell. That is, $\lambda=\left(\lambda_{1}, \ldots, \lambda_{\ell}\right)$ is a shifted rectangle if and only if $\lambda_{i}=\lambda_{1}-i+1$ for all $1 \leqslant i \leqslant \ell$. Note that shifted staircases are shifted rectangles.

Corollary 6.4. Let $\lambda^{\mathrm{dg}}$ (resp. $\lambda^{\mathrm{sh}}$ ) be a (shifted) rectangle and fix a column $\mathrm{c}$ of $\lambda^{\mathrm{dg}}$ (resp. $\left.\lambda^{\mathrm{sh}}\right)$. Then the number of horizontal (alt. vertical) adjacencies in column $c<\lambda_{1}$ (alt. row $r<\ell$ ) is equal to $f_{\lambda}$ (resp. $f_{\lambda}^{\text {sh }}$ ).

Proof. This a special case of Theorem 6.3.

The below result, in turn, is a corollary of Corollary 6.4.

COROLlary 6.5. The expected number of horizontal (resp. vertical) adjacencies in column $c<\lambda_{1}$ (resp. row $r<\ell$ ) of a uniformly random (shifted) rectangle $S Y T$ is equal to 1.

Proof. Apply Corollary 6.4, the definition of expectation, and the fact that each tableau occurs with the same probability.

Corollary 6.5 finally yields the following.

COROLlaRY 6.6. The expected number of adjacencies in a random 132-avoiding sorting network of length $N$ is $2(n-2)$.

Proof. This is a consequence of Corollary 6.5 by linearity of expectation and Proposition 6.2, since shifted staircases are shifted rectangles.

Compare this with the result of Schilling et al. below.

THeOREM 6.7 ([36, Thm. 1.3]). The expected number of patterns of the form $w_{k} w_{k+1} w_{k+2}=i(i+1) i$ for some $i \in[n-1]$ in a random 132-avoiding sorting network of length $N$ is 1 .

We note that for some (shifted) rectangles the variance of the total number of horizontal (or vertical) adjacencies seems to be an integer.

CONJECTURE 6.8. Let $\lambda=(n)^{n}$. The variance of the number of horizontal (alt. vertical) adjacencies in a SYT of shape $\lambda^{\mathrm{dg}}$ is $n-1$. The variance of the number of horizontal (alt. vertical) adjacencies in a shifted SYT of shape $\Delta_{n}^{\text {sh }}$ is $n-3$.

By Proposition 6.2, this would give the variance of the number of $i \in[n-1]$ such that $w_{i+1}=w_{i}+1$ (alternatively, $w_{i+1}=w_{i}-1$ ) in a random 132-avoiding sorting network $w$ of length $N$. In the same vein, Reiner has a conjecture [29, Conj. 3] on the variance of the number of $i(i+1) i, 1 \leqslant i \leqslant n-1$, in a random sorting network of length $N$, namely that it is $\frac{N-4}{N-2}$ for $n \geqslant 4$.

Some other corollaries of Theorem 6.3 are listed next. 
Corollary 6.9. Let $\lambda$ be a strict partition. Then the number of horizontal adjacencies in shifted $S Y T$ of shape $\lambda^{\text {sh }}$ in column $c<\ell(\lambda)$ is equal to $f_{\lambda}^{\text {sh }}$.

Proof. This is a special case of Theorem 6.3. Note that in a shifted SYT of shape $\lambda^{\text {sh }}$ the largest entry must always lie in column $\ell(\lambda)$ or greater.

The result below follows from Corollary 6.9 in the same way as Corollary 6.5 from Corollary 6.4.

Corollary 6.10. For any strict partition $\lambda$, the expected number of horizontal adjacencies in column $c<\ell(\lambda)$ of a random shifted SYT of shape $\lambda^{\text {sh }}$ is 1 .

Our final corollary may be obtained from Corollary 6.4 by noting that the left-hand side in both equations counts the total number of horizontal adjacencies. Note that both could also be written in terms of vertical adjacencies, that is $\{u, \mathbf{s} u\}$. Recall the definitions of $\square \backslash \nu$ and $\Delta_{n} \backslash \nu$ above Lemma 3.2.

Corollary 6.11. Let $\square=\left(n^{m}\right)$ be a rectangle. Then

$$
\sum_{\mu^{\mathrm{dg}} \subseteq \nu^{\mathrm{dg}} \subseteq \square \mathrm{dg}} f_{\mu} \cdot f_{\square \backslash \nu}=(n-1) f_{\square},
$$

where the sum is taken over all Young diagrams such that $\nu^{\mathrm{dg}}=\mu^{\mathrm{dg}} \cup\{u, \mathbf{e} u\}$ for some cell $u \in \square^{\mathrm{dg}} \backslash \mu^{\mathrm{dg}}$. Similarly,

$$
\sum_{\mu^{\mathrm{sh} \subseteq} \nu^{\mathrm{sh}} \subseteq \Delta_{n}^{\mathrm{sh}}} f_{\mu}^{\mathrm{sh}} \cdot f_{\Delta_{n} \backslash \nu}^{\mathrm{sh}}=(n-2) f_{\Delta_{n}}^{\mathrm{sh}},
$$

where the sum is taken over all shifted Young diagrams such that $\nu^{\mathrm{sh}}=\mu^{\mathrm{sh}} \cup\{u, \mathbf{e} u\}$ for some cell $u \in \Delta_{n}^{\mathrm{sh}} \backslash \mu^{\mathrm{sh}}$.

Compare (22) to the identities

$$
\sum_{\mu^{\mathrm{sh}} \subseteq \nu^{\mathrm{sh}} \subseteq \Delta_{n}^{\mathrm{sh}}} f_{\mu}^{\mathrm{sh}} \cdot f_{\Delta_{n} \backslash \nu}^{\mathrm{sh}}=f_{\Delta_{n}}^{\mathrm{sh}}
$$

where the sum is taken over all shifted Young diagrams such that $\nu^{\text {sh }}=\mu^{\text {sh }} U$ $\{u, \mathbf{e} u, \mathbf{e s} u\}$ for some cell $u \in \Delta_{n}^{\text {sh }} \backslash \mu^{\text {sh }}$, and

$$
\sum_{\mu^{\mathrm{sh}} \subseteq \nu^{\mathrm{sh}} \subseteq \Delta_{n}^{\mathrm{sh}}} f_{\mu}^{\mathrm{sh}} \cdot f_{\Delta_{n} \backslash \nu}^{\mathrm{sh}}=\left(\begin{array}{l}
n \\
2
\end{array}\right) f_{\Delta_{n}}^{\mathrm{sh}},
$$

where the sum is taken over all shifted Young diagrams such that $\nu^{\text {sh }}=\mu^{\text {sh }} \cup\{u\}$ for some cell $u \in \Delta_{n}^{\mathrm{sh}} \backslash \mu^{\mathrm{sh}}$. The first identity follows from the result in [36] mentioned above and the second is trivially true.

Open PRoBlem 6.12. Can equations (22), (23) and (24) be generalised?

If $w=s_{i} v$ is a reduced word for the reverse permutation in $\mathfrak{S}_{n}$, then so is $v s_{n-i-1}$. Thus we know that the probability of an adjacency is the same at every position in a random sorting network. This implies that the expected number of adjacencies before any position in a random sorting network grows linearly. Experiments suggest that the distribution of adjacencies converges also in probability to the uniform distribution, see Figure 21. Also the number of adjacencies in a 132-avoiding sorting network seems to grow in a nice manner.

CONJECture 6.13. Let $Y_{n}(\alpha)$ and $X_{n}(\alpha)$ denote the number of adjacencies $1 \leqslant k<$ $\alpha\left(\begin{array}{c}n \\ 2\end{array}\right)$ in a random sorting network and in a random 132-avoiding sorting network of size $n$ respectively. 

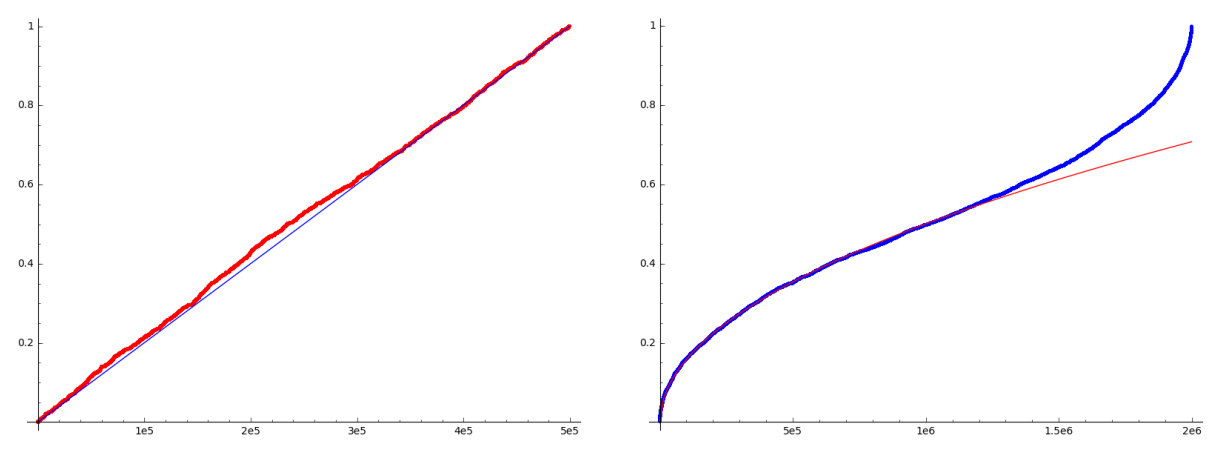

FiguRE 21. The number of adjacencies in an initial segment of a random sorting network of size $n=1000$, (left) seems to grow linearly. The number of adjacencies in an initial segment of a random 132avoiding sorting network of size $n=2000$ (right) seems to grow like a square root for the first half.

Then $Y_{n}(\alpha) / \mathbb{E}\left[Y_{n}(1)\right]$ converges in probability to $c \alpha$ for some constant $c$ and

$$
\lim _{n \rightarrow \infty} \mathbb{P}\left(\max _{0 \leqslant \alpha \leqslant 1}\left|X_{n}(\alpha) /(2(n-2))-g(\alpha)\right|>\epsilon\right)=0,
$$

where

$$
g(\alpha)= \begin{cases}\sqrt{\frac{\alpha}{2}} & \text { if } \alpha \in\left[0, \frac{1}{2}\right], \\ 1-\sqrt{\frac{1-\alpha}{2}} & \text { if } \alpha \in\left[\frac{1}{2}, 1\right] .\end{cases}
$$

As an interesting problem for further study, we would also like to mention the following.

OPEN PROBLEM 6.14. What is the behaviour of $\left|w_{k}-w_{k+1}\right|$ for $k \in[N-1]$ in random sorting networks $w$ of length $N$ ? See Figure 22.
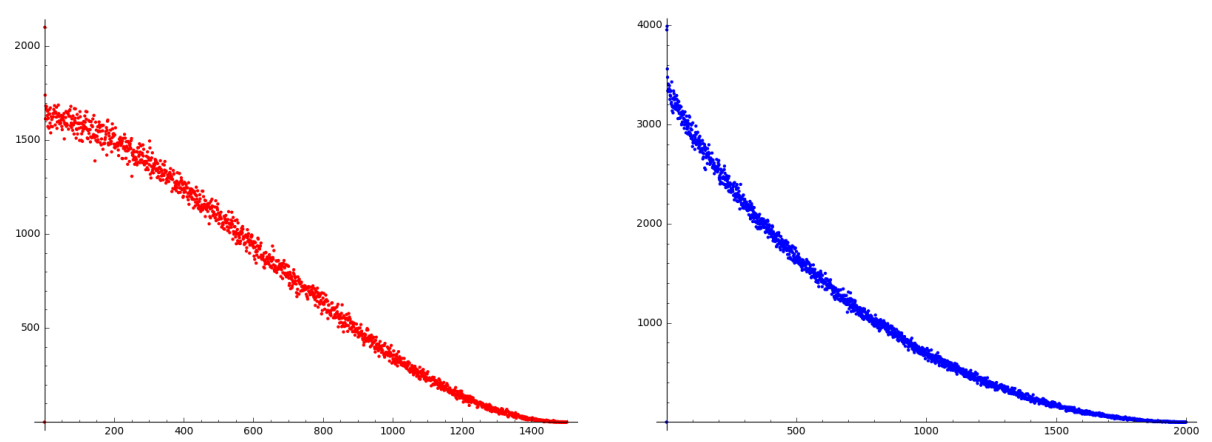

FiguRE 22. The number of occurrences of distances between adjacent entries in a random sorting network of size $n=1500$ (left) and a random 132-avoiding sorting network of size $n=2000$ (right).

For example, if $\left\{w_{k}, w_{k+1}\right\}=\{j, j+2\}$ in a 132-avoiding sorting network, then $k$ and $k+1$ are diagonally adjacent in the corresponding shifted SYT $Q_{w}$. 
Acknowledgements. The authors thank Dan Romik and John Stembridge for informative correspondences. This paper benefited from experimentation with Sage [35] and its combinatorics features developed by the Sage-Combinat community [34]. We are also grateful to the anonymous referees for helping us improve the paper.

\section{REFERENCES}

[1] Omer Angel, Alexander E. Holroyd, Dan Romik, and Bálint Virág, Random sorting networks, Adv. Math. 215 (2007), no. 2, 839-868.

[2] Philippe Biane, Representations of symmetric groups and free probability, Adv. Math. 138 (1998), no. 1, 126-181.

[3] Anders Björner and Michelle L. Wachs, Shellable nonpure complexes and posets. II, Trans. Amer. Math. Soc. 349 (1997), no. 10, 3945-3975.

[4] Duncan Dauvergne, The Archimedean limit of random sorting networks, https://arxiv.org/ abs/1802.08934, 2018.

[5] Robert Davis and Bruce Sagan, Pattern-avoiding polytopes, European J. Combin. 74 (2018), 48-84.

[6] Paul Edelman and Curtis Greene, Balanced tableaux, Adv. in Math. 63 (1987), no. 1, 42-99.

[7] Sergi Elizalde and Yuval Roichman, Arc permutations, J. Algebraic Combin. 39 (2014), no. 2, 301-334.

[8] Ilse Fischer, A bijective proof of the hook-length formula for shifted standard tableaux, https: //arxiv.org/abs/math/0112261, 2001.

[9] Susanna Fishel and Luke Nelson, Chains of maximum length in the Tamari lattice, Proc. Amer. Math. Soc. 142 (2014), no. 10, 3343-3353.

[10] Philippe Flajolet and Robert Sedgewick, Analytic combinatorics, Cambridge Univ. Press, Cambridge, 2009.

[11] James S. Frame, Gilbert de B. Robinson, and Robert M. Thrall, The hook graphs of the symmetric group, Canad. J. Math. 6 (1954), 316-325.

[12] Emden R. Gansner, Matrix correspondences and the enumeration of plane partitions, Ph.D. thesis, MIT, 1978.

[13] Curtis Greene, Albert Nijenhuis, and Herbert S. Wilf, A probabilistic proof of a formula for the number of Young tableaux of a given shape, Adv. in Math. 31 (1979), no. 1, 104-109.

[14] Mark D. Haiman, On mixed insertion, symmetry, and shifted Young tableaux, J. Combin. Theory Ser. A 50 (1989), no. 2, 196-225.

[15] _ Dual equivalence with applications, including a conjecture of Proctor, Discrete Math. 99 (1992), no. 1-3, 79-113.

[16] Vladimir Ivanov, Plancherel measure on shifted Young diagrams, in Representation Theory, Dynamical Systems, and Asymptotic Combinatorics, Amer. Math. Soc. Transl. Ser. 2, vol. 217, Amer. Math. Soc., Providence, RI, 2006, pp. 73-86.

[17] Sergey Kitaev, Patterns in permutations and words, Monographs in Theoretical Computer Science. An EATCS Series, Springer, Heidelberg, 2011.

[18] Witold Kraśkiewicz, Reduced decompositions in hyperoctahedral groups, C. R. Acad. Sci. Paris Sér. I Math. 309 (1989), no. 16, 903-907.

[19] —_ Reduced decompositions in Weyl groups, European J. Combin. 16 (1995), no. 3, 293313.

[20] Svante Linusson and Samu Potka, Properties of the Edelman-Greene bijection, J. Comb. 11 (2020), no. 2, 249-273.

[21] Ian G. Macdonald, Symmetric functions and Hall polynomials, 2nd ed., Oxford Mathematical Monographs, Oxford University Press, Oxford, 1995.

[22] Sho Matsumoto and Piotr Śniady, Random strict partitions and random shifted tableaux, Selecta Math. (N.S.) 26 (2020), no. 1, 10.

[23] Hiroshi Naruse and Soichi Okada, Skew hook formula for d-complete posets via equivariant K-theory, Algebr. Comb. 2 (2019), no. 4, 541-571.

[24] Jean-Cristophe Novelli, Igor Pak, and Alexander V. Stoyanovskii, A direct bijective proof of the hook-length formula, Discrete Math. Theor. Comput. Sci. 1 (1997), no. 1, 53-67.

[25] OEIS Foundation Inc., The On-Line Encyclopedia of Integer Sequences, 2020, http://oeis.org.

[26] Greta Panova and Piotr Śniady, Skew Howe duality and random rectangular Young tableaux, Algebr. Comb. 1 (2018), no. 1, 81-94.

[27] Boris Pittel and Dan Romik, Limit shapes for random square Young tableaux, Adv. in Appl. Math. 38 (2007), no. 2, 164-209. 
[28] Robert A. Proctor, Dynkin diagram classification of $\lambda$-minuscule Bruhat lattices and of dcomplete posets, J. Algebraic Combin. 9 (1999), no. 1, 61-94.

[29] Victor Reiner, Note on the expected number of Yang-Baxter moves applicable to reduced decompositions, European J. Combin. 26 (2005), no. 6, 1019-1021.

[30] Dan Romik, The surprising mathematics of longest increasing subsequences, Institute of Mathematical Statistics Textbooks, Cambridge University Press, New York, 2015.

[31] Bruce E. Sagan, On selecting a random shifted Young tableau, J. Algorithms 1 (1980), no. 3, $213-234$.

[32] Shifted tableaux, Schur Q-functions, and a conjecture of R. Stanley, J. Combin. Theory Ser. A 45 (1987), no. 1, 62-103.

[33] - The symmetric group. Representations, combinatorial algorithms, and symmetric functions, Graduate Texts in Mathematics, vol. 203, Springer-Verlag, New York, 2001.

[34] Sage-Combinat community, Sage-Combinat: enhancing Sage as a toolbox for computer exploration in algebraic combinatorics, 2008, http://combinat.sagemath.org.

[35] Sage Developers, Sagemath, the Sage Mathematics Software System Version 9.1, 2020, http: //www.sagemath.org.

[36] Anne Schilling, Nicolas M. Thiéry, Graham White, and Nathan Williams, Braid moves in commutation classes of the symmetric group, European J. Combin. 62 (2017), 15-34.

[37] Marcel-Paul Schützenberger, Quelques remarques sur une construction de Schensted, Math. Scand. 12 (1963), no. 1, 117-128.

[38] Richard P. Stanley, Enumerative combinatorics. Vol. 2, Cambridge Studies in Advanced Mathematics, vol. 62, Cambridge University Press, Cambridge, 1999.

[39] , Promotion and evacuation, Electron. J. Combin. 16 (2009), no. 2, The Björner Festschrift volume, 9 (24 pages).

[40] John R. Stembridge, Shifted tableaux and the projective representations of symmetric groups, Adv. Math. 74 (1989), no. 1, 87-134.

[41] - On the of fully commutative elements of Coxeter groups, J. Algebraic Combin. 5 (1996), no. 4, 353-385.

[42] — The enumeration of fully commutative elements of Coxeter groups, J. Algebraic Combin. 7 (1998), no. 3, 291-320.

[43] Bridget E. Tenner, On the expected number of commutations in reduced words, Australas. J. Combin. 62 (2015), no. 1, 147-154.

[44] Robert M. Thrall, A combinatorial problem, Michigan Math. J. 1 (1952), no. 1, 81-88.

Svante Linusson, KTH Royal Institute of Technology, Department of Mathematics, Stockholm, Sweden

E-mail : linusson@kth.se

Samu PotKa, KTH Royal Institute of Technology, Department of Mathematics, Stockholm, Sweden E-mail : potka@kth.se

Robin SulzGruber, KTH Royal Institute of Technology, Department of Mathematics, Stockholm, Sweden

E-mail : robinsul@kth.se 